\title{
Lattice Boltzmann simulations of low-Reynolds-number flows past fluidized spheres: effect of inhomogeneities on the drag force
}

\author{
Gregory J. Rubinstein ${ }^{1}$, Ali Ozel ${ }^{1}$, Xiaolong Yin², J. J. Derksen ${ }^{3}$ and Sankaran \\ Sundaresan $^{1} \dagger$ \\ ${ }^{1}$ Department of Chemical and Biological Engineering, Princeton University, Princeton, NJ 08540, USA \\ ${ }^{2}$ Department of Petroleum Engineering, Colorado School of Mines, Golden, CO 80401, USA \\ ${ }^{3}$ School of Engineering, University of Aberdeen, Aberdeen AB24 3UE, United Kingdom
}

(Received xx; revised xx; accepted xx)

The formation of inhomogeneities within fluidized beds, both in terms of the particle configurations and flow structures, have a pronounced effect on the interaction force between the fluid and particles. While recent numerical studies have begun to probe the effects of inhomogeneities on the drag force at the particle scale, the applicability of prior micro-scale constitutive drag relations is still limited to random, homogeneous distributions of particles. Since an accurate model for the drag force is needed to predict the fluidization behavior, the current study utilizes the lessons of prior inhomogeneity studies in order to derive a robust drag relation that is both able to account for the effect of inhomogeneities and applicable as a constitutive closure to larger-scale fluidization simulations. Using fully-resolved lattice Boltzmann simulations of systems composed of fluid and monodisperse spherical particles in the low-Reynolds-number $(R e)$ regime, the fluid-particle drag force, normalized by the ideal Stokes drag force, is found to significantly decrease, over a range of length scales, as the extent of inhomogeneities increases. The extent of inhomogeneities is found to most effectively be quantified through one of two subgrid-scale quantities: the scalar variance of the particle volume fraction or the drift flux, which is the correlation between the particle volume fraction and slip velocity. Scale-similar models are developed to estimate these two sub-grid measures over a wide range of system properties. Two new drag constitutive models are proposed that are not only functions of the particle volume fraction and the Stokes number $(S t)$, but also dependent on one of these sub-grid measures for the extent of inhomogeneities. Based on the observed, appreciable effect of inhomogeneities on drag, these new low $R e$ drag models represent a significant advancement over prior constitutive relations.

Key words:

$\dagger$ Email address for correspondence: sundar@ princeton.edu 


\section{Introduction}

The ability to attain quantitatively reliable predictions of fluidized bed behavior is highly dependent on the accuracy of the fluid-particle drag force, since in fluidized beds, the buoyant weight of particles is principally balanced by the drag force exerted by the flowing fluid. In numerical studies of industrial-scale systems, the interactions between fluid and particles cannot be fully resolved at the scale of the particle's surface due to limitations in computational power. Instead, in these large-scale simulations, the fluid computational grid size is larger than a particle diameter, and so constitutive relations for the fluid-particle drag force are necessary. The accuracy of these drag models at the particle-scale level is vital in order to achieve quantitative precision in the study of fluidized beds. Despite this fact, there are a number of limitations to the drag models that are commonly employed in the literature. While a number of recent studies (Zhou et al. 2014; Wang et al. 2011; Xu et al. 2007) have elucidated the effect of the magnitude and direction of inhomogeneities in the particle configuration on the drag, these studies have not resulted in a generalized inhomogeneity-dependent drag model that can be applied as a constitutive closure to larger-scale fluidization simulations. Thus, the current work primarily addresses the need for the development of such a drag relation that accounts for inhomogeneities in the distribution of particles.

Prior drag models that have been employed in large-scale simulations of fluidized beds are typically based on either the sedimentation of particles (Richardson \& Zaki 1954; Wen \& Yu 1966; Garside \& Al-Dibouni 1977) or fixed particle beds (Hill et al. 2001; van der Hoef et al. 2005; Beetstra et al. 2007; Tenneti et al. 2011). Typically, these drag models are expressed in terms of a dimensionless drag force, $F$, which is defined as:

$$
F=\frac{\boldsymbol{F}_{f p} \cdot \boldsymbol{u}_{\text {slip }}}{\boldsymbol{F}_{d, \text { Stokes }} \cdot \boldsymbol{u}_{\text {slip }}}
$$

where $\boldsymbol{F}_{f p}$ is the total fluid-particle interaction force per particle minus the generalized buoyancy arising from the slowly varying stress field, $\boldsymbol{u}_{\text {slip }}$ is the slip velocity, which is the difference between the fluid velocity, $\boldsymbol{u}_{f}$, and the particle velocity, $\boldsymbol{v}_{p}$, and the Stokes drag relation on a single spherical particle at infinite dilution in a viscous fluid is given by:

$$
\boldsymbol{F}_{d, \text { Stokes }}=3 \pi \mu_{f} d_{p} \boldsymbol{u}_{\text {slip }},
$$

where $d_{p}$ is diameter of the particle and $\mu_{f}$ is the dynamic fluid viscosity. These drag models are typically expressed as functions of the particle volume fraction, $\phi$, and Reynolds number $(R e)$, which is defined as:

$$
R e=\frac{\rho_{f}(1-\phi)\left|\boldsymbol{u}_{\text {slip }}\right| d_{p}}{\mu_{f}},
$$

where $\rho_{f}$ is the fluid density. Recently, the Rubinstein et al. (2016) model expanded upon these traditional drag relations by accounting for the effects of particle inertia through the Stokes number $(S t)$, which is the ratio of the particle relaxation time to the fluid relaxation time, and is defined as:

$$
S t=\frac{\rho_{p}(1-\phi)\left|\boldsymbol{u}_{s l i p}\right| d_{p}}{18 \mu_{f}} .
$$

While the Rubinstein et al. (2016) drag model provides a significant improvement over prior drag relations in its ability to implicitly account for the effects of particle translation and rotation through $S t$, one key limitation that persists is that this drag model is only applicable to random, 
homogeneous distributions of particles. This constitutive relation relies on the key assumption that the distribution of particles is homogeneous at the scale at which the model is applied. However, the effect of the extent of inhomogeneities on the drag force has been found to be significant, even at the small scale of several particle diameters (Kriebitzsch et al. 2013; Zhou et al. 2014). Thus, the goal of the current work is to develop a new drag model that is able to account for the effect of inhomogeneities.

The importance of inhomogeneities in the particle distribution on the behavior of fluidized beds has been demonstrated by a number of studies. In fluidized bed simulations based on the two-fluid model (TFM), achieving grid size convergence with traditional drag closures has proven to be very difficult, even at grid sizes as small as $10 d_{p}$, due to the presence of heterogeneous structures (Agrawal et al. 2001; Igci et al. 2008; Li et al. 2014). In particular, for Geldart Group A particles, typical fine-grid TFM simulations are unable to predict the behavior that is observed experimentally (Cloete et al. 2015; Liu et al. 2015; Wang et al. 2009; Lu et al. 2009). These shortcomings are especially pronounced in high-velocity systems, where homogeneous drag models are unable to resolve all of the mesoscale structures (Hong et al. 2016). While the effects of particle cohesion and fluid turbulence could have contributed to the observed differences between the TFM simulations and experiments of fluidized beds with Geldart Group A particles, prior studies, like that of Wang et al. (2009), have found that, due to the significance of inhomogeneities at relatively small length scales, a sufficiently fine grid size is needed in order to accurately simulate the flow characteristics using TFM. Thus, failing to account for particle structures significantly affects the computed fluid-particle drag. In the current work, by focusing on low $R e$ systems with non-cohesive particles, we singularly study the effects of inhomogeneities. This choice of fluidized bed simulations therefore provides a direct method to probe the specific effects of inhomogeneities on the drag force.

One of the key measures that has been used in prior studies to quantify the extent of inhomogeneities is the solid volume fraction gradient, $\nabla \phi$. This quantity, $\nabla \phi$, has been found to correlate with the grid size required to achieve grid convergence (Fullmer \& Hrenya 2016). Furthermore, in fixed particle systems with simple linear variations in $\phi$, it has been demonstrated that the drag is affected by both the magnitude of $\boldsymbol{\nabla} \phi$ and the angle between $\boldsymbol{u}_{\text {slip }}$ and $\boldsymbol{\nabla} \phi$ (Zhou et al. 2014; Li et al. 2016). Larger magnitudes of $\nabla \phi$ lead to larger changes in the drag. In terms of the directional effect, when $\nabla \phi$ is aligned with the slip velocity, the fluid flow has to move through an area where there is a high concentration of particles, causing for there to be an increase in the drag. On the other hand, when $\nabla \phi$ is perpendicular to the slip velocity, the fluid flow can easily by-pass the areas with high concentration of particles, leading to a reduction in the drag. Thus, the drag is maximized when $\boldsymbol{u}_{\text {slip }}$ is parallel to $\boldsymbol{\nabla} \phi$, and is minimized when $\boldsymbol{u}_{\text {slip }}$ is perpendicular to $\nabla \phi$ (Zhou et al. 2014; Li et al. 2016). In demonstrating the importance of the $\nabla \phi$ measure, these studies have further emphasized how significant inhomogeneities are to the behavior of fluidized beds.

While there have been some prior attempts to correct the homogeneous drag models with additional terms that are computed using detailed particle distribution information (Wang et al. 2011; Xu et al. 2007), prior studies have not arrived at a drag model that can both account for the effect of inhomogeneities and be easily applied to TFM and computational fluid dynamicsdiscrete element method (CFD-DEM) larger-scale simulations. The goal of the current study is to therefore take the insights gained from these prior studies, and use them to derive a new drag constitutive relation that not only accounts for the effect of particle distribution inhomogeneities, but is also applicable as a closure to a variety of larger-scale simulations. Towards this goal, lattice Boltmann method (LBM) simulations of fluidized beds are performed. Using the detailed flow and particle configuration data obtained from these simulations, the fluid-particle drag is found 


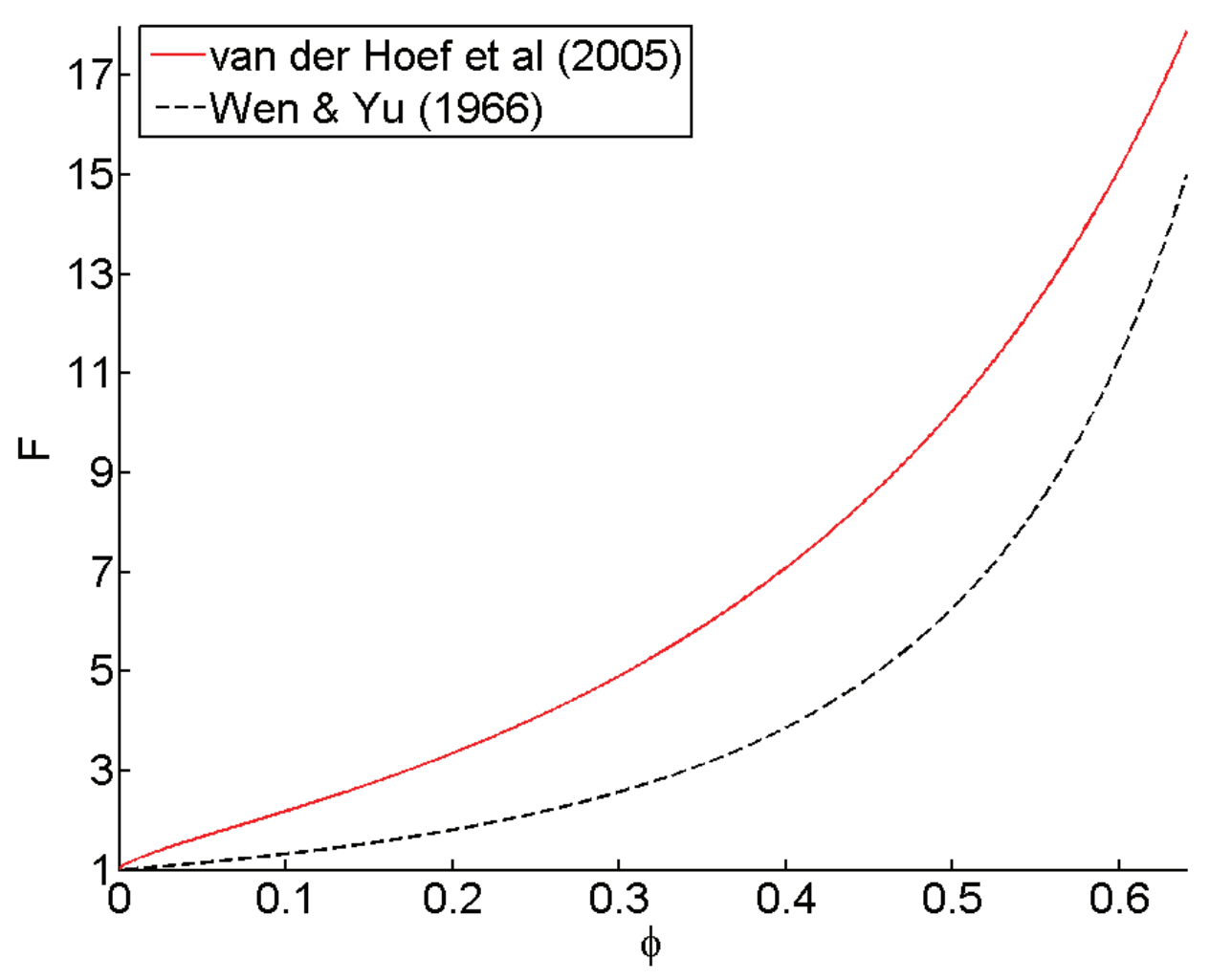

FIgURE 1. The low Re dimensionless drag force, $F$, is plotted as a function of particle volume fraction, $\phi$, for the both the van der Hoef et al. (2005) model for fixed particle beds (high St limit) and the Wen \& Yu (1966) model for sedimenting particle beds (low $S t$ limit).

to depend on a sub-grid-scale measure for the extent of inhomogeneities. Models to estimate these sub-grid quantities are developed that are based on a scale-similarity approach, which was originally derived for single-phase turbulence (Germano et al. 1991) and later adapted to applications in multi-phase flow (Parmentier et al. 2012; Ozel et al. 2013). By accounting for the effect of inhomogeneities, these newly-derived constitutive relations for the fluid-particle drag force provide a significant improvement over prior models that assumed a random, homogeneous distribution of particles.

In section 2, we describe prior drag modeling attempts, our LBM simulation scheme, and the filtering method used to analyze the results. In section 3 , we provide the simulation results from this study, as well as the development of our new drag models that account for inhomogeneities in the distribution of particles in low Re systems. In section 4, we provide some concluding remarks on the overall findings of this study.

\section{Methodology}

\subsection{Prior drag modeling}

In Figure 1, we see that in the low Re regime, the fixed bed dimensionless drag (van der Hoef et al. 2005) is significantly greater than the particle sedimentation drag (Wen \& Yu 1966) over the entire range of $\phi$. While the granular temperature has been shown to have a significant effect on the drag in dynamic fluidized beds at larger Re (Tang et al. 2016), this quantity has a small effect in the low Re regime. Instead, this large difference in the drag relations, which was identified in our prior work (Rubinstein et al. 2016), is attributed to $S t$ (equation 1.4). In the low Re regime, the fixed bed drag can be thought of as the high-St-limit drag, while the particle sedimentation 


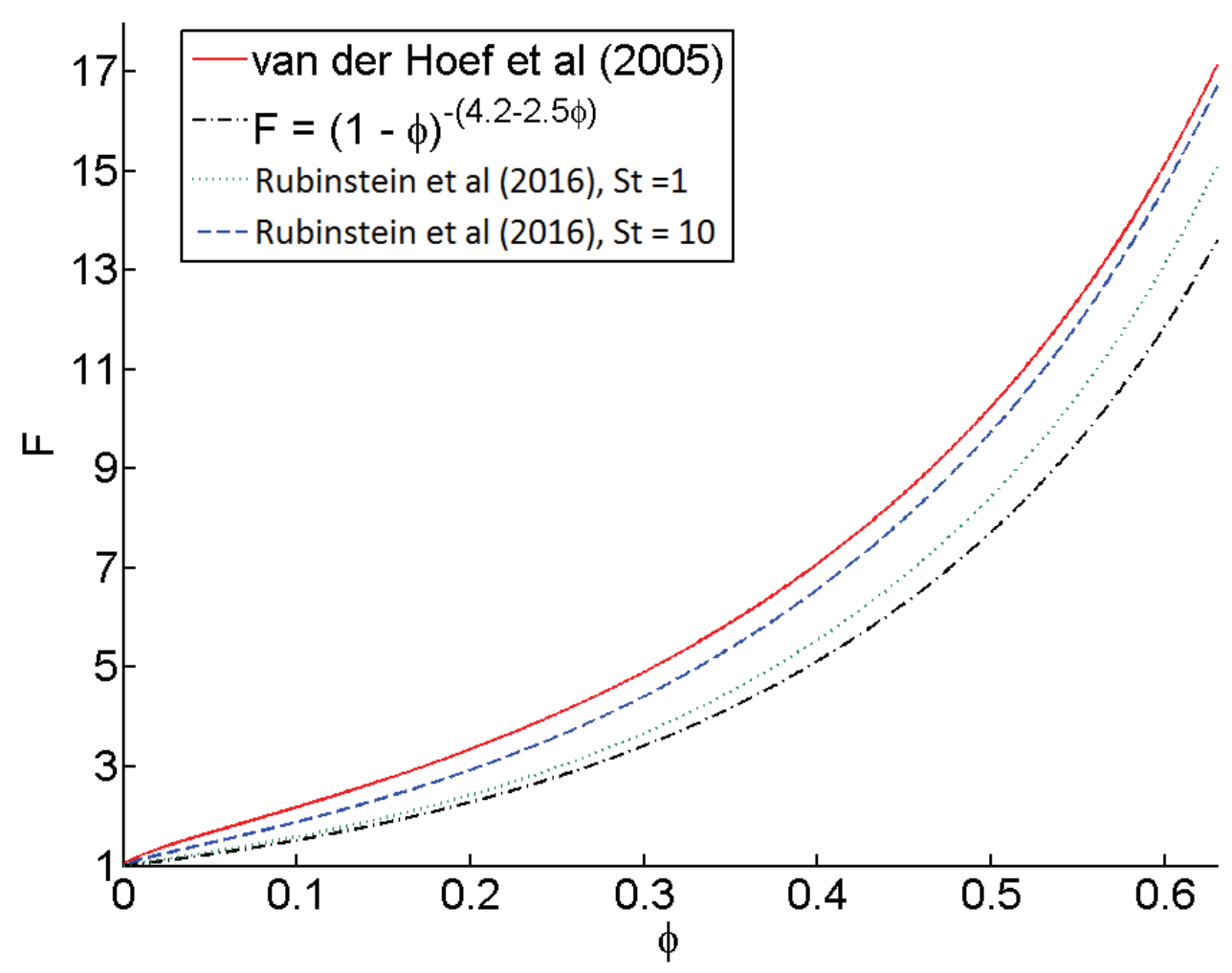

FIGURE 2. The Rubinstein et al. (2016) drag model, which accounts for the effects of St, is plotted over a range of $\phi$ and St. The van der Hoef et al. (2005) curve represents the high St limit of this model, while the $F=(1-\phi)^{-(4.2-2.5 \phi)}$ curve represents the low St limit of this model.

drag can be thought of as the low-St-limit drag. Furthermore, in this prior work, we proposed a new $S t$-dependent drag model that was able to bridge the transition from the low $S t$ limit to the high St limit. This drag model has the following form:

$$
F(\phi, S t)=\alpha\left(S t_{\text {mod }}\right) F_{v d H}(\phi)+\left(1-\alpha\left(S t_{\text {mod }}\right)\right)(1-\phi)^{-(n(\phi)-2)},
$$

where the high-St-limit drag is given by the van der Hoef et al. (2005) curve:

$$
F_{v d H}(\phi)=\frac{10 \phi}{1-\phi}+(1-\phi)^{3}(1+1.5 \sqrt{\phi})
$$

and the low-St-limit drag is given by:

$$
F_{\text {low } S t}(\phi)=(1-\phi)^{-(n(\phi)-2)},
$$

with $n(\phi)=6.2-2.5 \phi$. The parameter $\alpha$, which describes the transition from the low-St limit to the high-St limit, is given by:

$$
\alpha\left(S t_{m o d}\right)=\frac{1}{2}\left(1+\frac{S t_{m o d}-10}{S t_{m o d}+10}\right)
$$

where the modified Stokes number, $S t_{\text {mod }}$, is defined as:

$$
S t_{\text {mod }}=\frac{S t}{(1-\phi)^{2}} .
$$

This new St-dependent drag model is shown in Figure 2. 


\subsection{LBM scheme}

In this study, LBM simulations of fluidized beds are employed in order to develop a new drag model that can account for the effect of inhomogeneities. In LBM, the fluid flow is represented by the movement of fluid parcels along a three-dimensional lattice of nodes. These fluid dynamics are governed by the Boltzmann equation, which is discretized in both time and space. In the low-Mach-number limit, these discretized Boltzmann equations converge to the incompressible Navier-Stokes equations when averaged over a sufficiently large volume (Chen \& Doolen 1998). The LBM scheme employed in this study is based on a slight variant of the widely used LBGK scheme (Qian et al. 1992). This scheme, which is used to solve for the evolution of the fluid density and momentum distributions, is described in more detail by Somers (1993) and Eggels \& Somers (1995).

The interactions between the solid particles and fluid, which were first studied using LBM by Ladd (1994), are resolved using a technique similar to the immersed boundary method (Goldstein et al. 1993; ten Cate et al. 2002; Derksen \& Van den Akker 1999). This forcing mechanism ensures that the no-slip boundary condition is satisfied by imposing additional forces on the fluid along the surface of the solid particle. The net force and torque that the fluid exerts on a solid particle is then computed by summing over these local surface forces. An additional particleparticle lubrication force, which accounts for unresolved, sub-grid flow contributions when the gap between two particles is smaller than the lattice grid size, is introduced into the scheme (Nguyen \& Ladd 2002; Kim \& Karilla 1991). Furthermore, in order to computationally facilitate collisions between particles, each of the solid particles is given a small degree of softness. Particle-particle collisions still effectively resemble hard sphere collisions, as the maximum overlap distance is about 0.001 particle diameters. At each time step, the velocity and position of the particles are updated by using the total force and torque acting on each particle. Further details of the LBM numerical scheme used in this study can be found in Derksen \& Sundaresan (2007) and Rubinstein et al. (2016).

In the current study, fluidized beds with spherical monodisperse particles are simulated in a cubic domain with fully periodic boundary conditions in all dimensions. In this periodic system, a body force is applied to all of the fluid and particles in order to drive flow in the system and balance the net weight of the fluid and particles (Derksen \& Sundaresan 2007). Since the lattice units are dimensionless, for simplicity, the lattice spacing, $\Delta$, and the time step are taken to be 1. The parameters that define a given system are: the particle diameter, $d_{p}$, the fluid kinematic viscosity, $v_{f}$, the particle-to-fluid density ratio, $\rho_{p} / \rho_{f}$, the strength of the applied external body force, $f_{\text {ext }}$, the total number of particles, $N$, and the dimensions of the lattice, $n_{x}, n_{y}$, and $n_{z}$. For the current study, $d_{p}=12$ and $v_{f}=0.1$. While larger values of $d_{p}$ would correspond to higher resolutions of the fluid flow profile, the chosen combination of values was previously shown to provide a sufficient level of resolution in order to achieve convergence in the flow behavior in the low Re regime (Rubinstein et al. 2016). Furthermore, in order to obtain a sufficiently large domain where inhomogeneous structures are able to form, we choose: $n_{x}=n_{y}=n_{z}=12 d_{p}$. The domain-averaged volume fraction, $\langle\phi\rangle$, is defined as:

$$
\langle\phi\rangle=\frac{N\left(\frac{\pi}{6} d_{p}^{3}\right)}{n_{x} n_{y} n_{z}} .
$$

St cannot be set a priori, and so it is, instead, computed from the results of the simulation. In this study, $f_{\text {ext }}, \rho_{p} / \rho_{f}$, and $\langle\phi\rangle$ are varied so that large ranges of $S t$ and inhomogeneous structures are sampled. In particular, since the study is focused on the low Re regime, a given simulation is defined by the following pair of parameters: $\rho_{p} / \rho_{f}$ and $\langle\phi\rangle$.

In this study, using the insights developed in our prior work (Rubinstein et al. 2016), we 
utilized two additional types of LBM simulations: high St limit and low St limit beds. Since particles are slow to adapt to the surrounding fluid in the high St regime, in the high St limit simulations, the fluid flow establishes an equilibrium around a bed of fixed particles, with the particle translational and rotational velocities held fixed at 0 . Since particles adapt very quickly to the surrounding fluid in the low $S t$ regime, in the low $S t$ limit simulations, the fluid flow and particle velocities are allowed to evolve until the net force and torque on each particle is 0 , while the particles are held fixed in place. Further details of the high $S t$ and low St limit simulations are presented in Rubinstein et al. (2016).

\subsection{Analyzing LBM results: Filtering procedure}

The goal of the current study is to analyze the effect of inhomogeneous structures on the fluid-particle drag. While our prior analysis simply looked to compute domain-averaged LBM quantities, such an approach would be insufficient for resolving these different structures. Thus, in the current study, a filtering technique is employed, in which the LBM simulation results are analyzed over a range of length scales. In this method, first of all, the Lagrangian particle variables are mapped onto an Eulerian grid with a base cell size of $\Delta_{b}=1 d_{p}$ using a Gaussiantype mapping function (Pepiot \& Desjardins 2012), $g(s)$, which is given by:

$$
g(s)=\left\{\begin{array}{ll}
\frac{1}{4} s^{4}-\frac{5}{8} s^{2}+\frac{115}{192}, & s \leqslant 0.5 \\
-\frac{1}{6} s^{4}+\frac{5}{6} s^{3}-\frac{5}{4} s^{2}+\frac{5}{24} s+\frac{55}{96}, & 0.5<s \leqslant 1.5 \\
\frac{(2.5-s)^{4}}{24}, & 1.5<s \leqslant 2.5 \\
0, & s>2.5
\end{array} .\right.
$$

In equation 2.7, $s$ is the distance between the particle center and grid center, normalized by the Eulerian grid size, $\Delta_{b}$. The fully-resolved fluid lattice grid variables are then coarsened, via phase Favre averaging, to the $\Delta_{b}=1 d_{p}$ Eulerian grid using the mapping function in equation 2.7. The size of the base Eulerian grid is chosen to be $1 d_{p}$, since below this length scale, the fluctuations in the structures are too fine to effectively model. At this base grid size, the particle volume fraction, fluid velocity, particle velocity, and fluid-particle interaction force per unit volume are denoted as $\phi, \boldsymbol{u}_{f}, \boldsymbol{v}_{p}$, and $\boldsymbol{f}_{f p}$, respectively. Once all of these variables have been computed over this $1 d_{p}$ Eulerian grid, the structures in each simulation system are studied over a range of length scales by defining a filter size, $\Delta_{f}$, which is greater than or equal to the base grid size, $\Delta_{b}$. Each of these variables is then filtered by performing either simple volume-averaging $\left(\phi, \boldsymbol{f}_{f p}\right)$ or Favre averaging $\left(\boldsymbol{u}_{f}, \boldsymbol{v}_{p}\right)$. These filtered variables are denoted as $\bar{\phi}, \tilde{\boldsymbol{u}}_{f}, \tilde{\boldsymbol{v}}_{p}$, and $\overline{\boldsymbol{f}}_{f p}$, and are computed as follows:

$$
\begin{gathered}
\bar{\phi}=\frac{\sum_{i} V_{c e l l, i} \phi_{i}}{\sum_{i} V_{c e l l, i}} \\
\tilde{u}_{f, j}=\frac{\sum_{i} V_{c e l l, i}\left(1-\phi_{i}\right) u_{f, i j}}{\sum_{i} V_{c e l l, i}\left(1-\phi_{i}\right)} \\
\tilde{v}_{p, j}=\frac{\sum_{i} V_{c e l l, i} \phi_{i} v_{p, i j}}{\sum_{i} V_{c e l l, i} \phi_{i}} \\
\bar{f}_{f p, j}=\frac{\sum_{i} V_{c e l l, i} f_{f p, i j}}{\sum_{i} V_{c e l l, i}}
\end{gathered}
$$

where each of these sums is performed over all of the base grid cells that are contained within a particular filter cell, $V_{c e l l, i}$ is the volume of the $i^{\text {th }}$ base grid cell, and the index $j$ is used to denote 
the $x, y$, and $z$ components. By using this filtering technique, the effect of inhomogeneities on the flow behavior is studied over a range of length scales. To put our prior domain-averaged analysis into context, it is equivalent to performing a filtering analysis with $\Delta_{f}=\Delta_{b}=n_{x}$.

\section{Simulation results}

\subsection{Effect of inhomogeneities on drag}

In order to demonstrate the significance of the effect of inhomogeneities on the drag force, we compared the drag in systems with random, homogeneous distributions of particles to those with inhomogeneous structures. Drawing upon our methodology in our prior work, our goal was to establish these inhomogeneity effects at the high and low $S t$ limits. In doing so, we are able to isolate the effect of inhomogeneities from that of $S t$.

Random, homogeneous configurations of particles are obtained using a thermalization technique via DEM simulations, which is described in further detail by Rubinstein et al. (2016). These random, homogeneous distributions are employed as the initial particle configurations in fluidized bed simulations. Inhomogeneous configurations of particles are then obtained by running fluidized bed simulations until a statistical steady state is reached. The statistical steady state is based on the time evolution of $\left\langle\boldsymbol{u}_{\text {slip }}\right\rangle$. The final, inhomogeneous configuration is not correlated with the initial, homogeneous configuration, and so the inhomogeneity-dependent drag results obtained in this study are unaffected by the thermalization technique. In our study, we find that fluidized beds with different values of $\rho_{p} / \rho_{f}$ induce different extents of inhomogeneities, and so we investigate the properties of inhomogeneous configurations originating from fluidized beds with a range of $\rho_{p} / \rho_{f}$ values.

In figure 3, the random, homogeneous drag results are compared with the inhomogeneous drag results at the (a) high $S t$ limit and (b) low St limit. In this figure, the dimensionless drag force, $F$, is plotted as a function of the filtered particle volume fraction, $\bar{\phi}$. In terms of filtered quantities, $F$ is defined as:

$$
F=\frac{\overline{\boldsymbol{F}}_{f p} \cdot \tilde{\boldsymbol{u}}_{\text {slip }}}{3 \pi \mu_{f} d_{p} \tilde{\boldsymbol{u}}_{\text {slip }} \cdot \tilde{\boldsymbol{u}}_{\text {slip }}},
$$

where $\tilde{\boldsymbol{u}}_{s l i p}=\tilde{\boldsymbol{u}}_{f}-\tilde{\boldsymbol{v}}_{p}$ and $\overline{\boldsymbol{F}}_{f p}=\left(V_{p} / \bar{\phi}\right) \overline{\boldsymbol{f}}_{f p}$. For the current analysis, all of these quantities are computed using a filter size of $\Delta_{f} / d_{p}=3$. In figure 3 , each drag curve is computed by binaveraging the $F$ values with $\bar{\phi}$, using a bin-width of 0.01 . In order to more simply visualize the effects of inhomogeneities over a large range of $\bar{\phi}$, each drag curve combines the results from systems with $\langle\phi\rangle=0.1,0.15,0.2,0.25$, and 0.3 .

As expected, in figure $3 \mathrm{a}$, the random, homogeneous, high $S t$ limit drag results (red line) converge to the homogeneous, high St limit curve (van der Hoef et al. 2005) given in equation 2.2. In figure $3 \mathrm{~b}$, the random, homogeneous, low $S t$ limit drag results (red line) nearly converge to the homogeneous, low St limit curve provided in equation 2.3 (Wen \& Yu (1966) type drag), with a modest observed difference due to the slight domain-size dependence of the homogeneous, low $S t$ drag. In figure 3a, in addition to the homogeneous curve, three inhomogeneous, high St limit drag curves are shown that are derived from inhomogeneous particle configurations arising from fluidized beds with $\rho_{p} / \rho_{f}=10,100$, and 300, respectively. These same set of inhomogeneous particle configurations are used to obtain the three inhomogeneous, low $S t$ limit drag curves shown in figure $3 b$.

From both figures $3 \mathrm{a}$ and $\mathrm{b}$, it is clear that there is a significant reduction in drag when moving from the homogeneous curve to the inhomogeneous curves at both the high and low $S t$ 


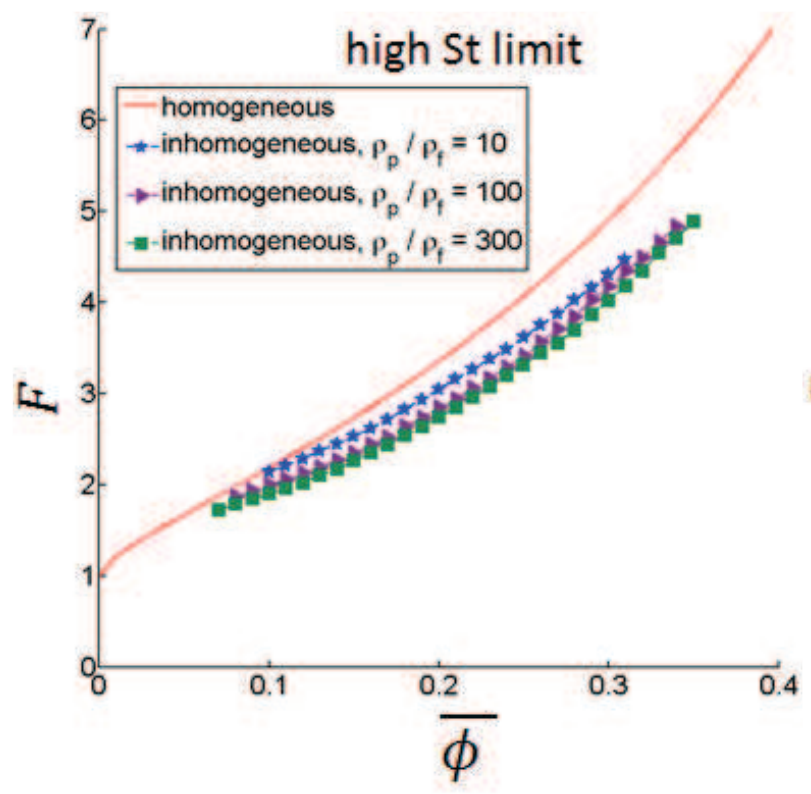

(a)

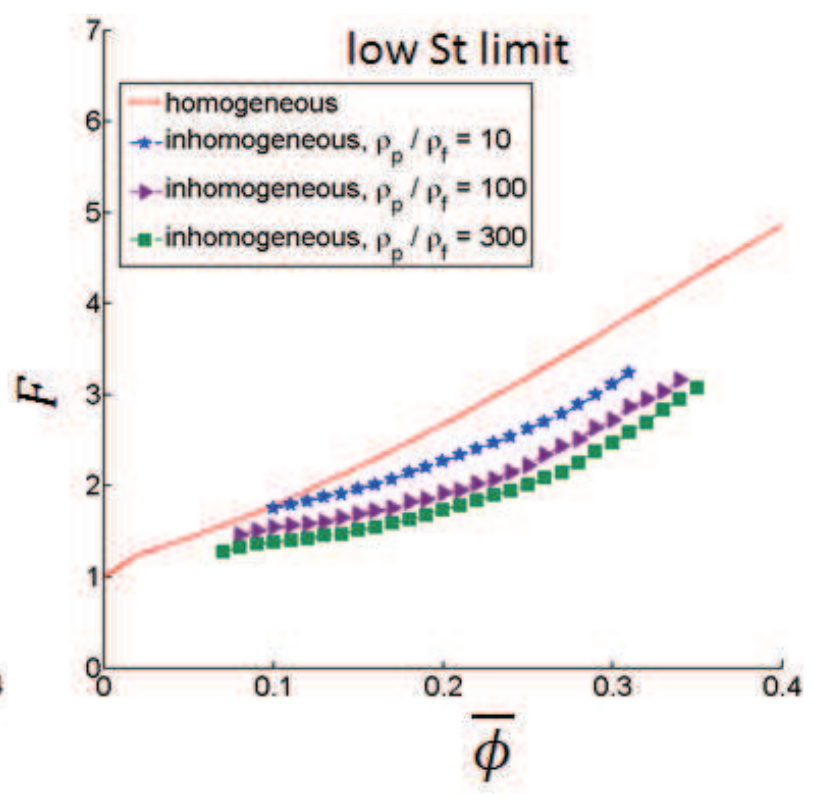

(b)

FIGURE 3. The dimensionless drag force, $F$, is plotted as a function of the filtered volume fraction, $\bar{\phi}$, for both randomly homogeneous and inhomogeneous particle configurations for (a) the high St limit and (b) low St limit. The inhomogeneous drag configurations are taken from fluidized systems with three differerent values of $\rho_{p} / \rho_{f}(10,100$, and 300). Each drag curve is computed by bin-averaging the drag with $\bar{\phi}$, using a bin-width of 0.01 and $\Delta_{f} / d_{p}=3$, and combines the results from systems over a range of $\langle\phi\rangle(0.1,0.15$, $0.2,0.25$, and 0.3$)$.

limits, with a stronger effect observed at the low St limit. The magnitude of this drag reduction increases for inhomogeneous configurations arising from fluidized beds with larger $\rho_{p} / \rho_{f}$. Larger values of $\rho_{p} / \rho_{f}$ result in a higher growth rate of inertial instabilities, leading to a greater extent of inhomogeneities in the configurations. Thus, since higher values of $\rho_{p} / \rho_{f}$ are associated with configurations with greater extents of inhomogeneities, in the low Re regime, increases in the extent of inhomogeneities result in decreases in the drag at both the high and low St limits. In terms of the $\bar{\phi}$-bin-averaged drag results, at $\bar{\phi}=0.3$, there is a roughly $18 \%$ reduction in drag when moving from the high $S t$ limit, homogeneous curve to the high St limit, inhomogeneous curve that is derived from particle configurations taken from fluidized beds with $\rho_{p} / \rho_{f}=300$, and this analogous reduction in drag is about $34 \%$ for the low $S t$ limit case. Thus, we conclude that homogeneous drag relations are insufficient in modeling the fluid-particle drag in inhomogeneous systems at both the high and low St limits.

In section 1 , the mechanism for the effect of inhomogeneities on the drag was interpreted in terms of $\boldsymbol{\nabla} \phi_{\|}$and $\boldsymbol{\nabla} \phi_{\perp}$, the components of $\boldsymbol{\nabla} \phi$ that are parallel and perpendicular to $\boldsymbol{u}_{\text {slip }}$, respectively. In this study, since the dominant effect of inhomogeneities is a reduction in drag, we conclude that the type of inhomogeneous structures that form within our fluidized bed simulations are ones for which $\boldsymbol{\nabla} \phi$ is primarily perpendicular to $\boldsymbol{u}_{\text {slip }}$, thus allowing the fluid to more easily by-pass the denser parts of these particle structures.

In our prior work (Rubinstein et al. 2016), we established that the fluidized bed drag over a full range of $S t$ can be determined from an interpolation of the high and low $S t$ limits. Thus, based on our conclusions at the high and low $S t$ limits, we are able to infer that homogeneous drag models are unable to properly describe the drag force in fluidized beds due to the significant reduction in drag caused by the presence of inhomogeneous structures. 


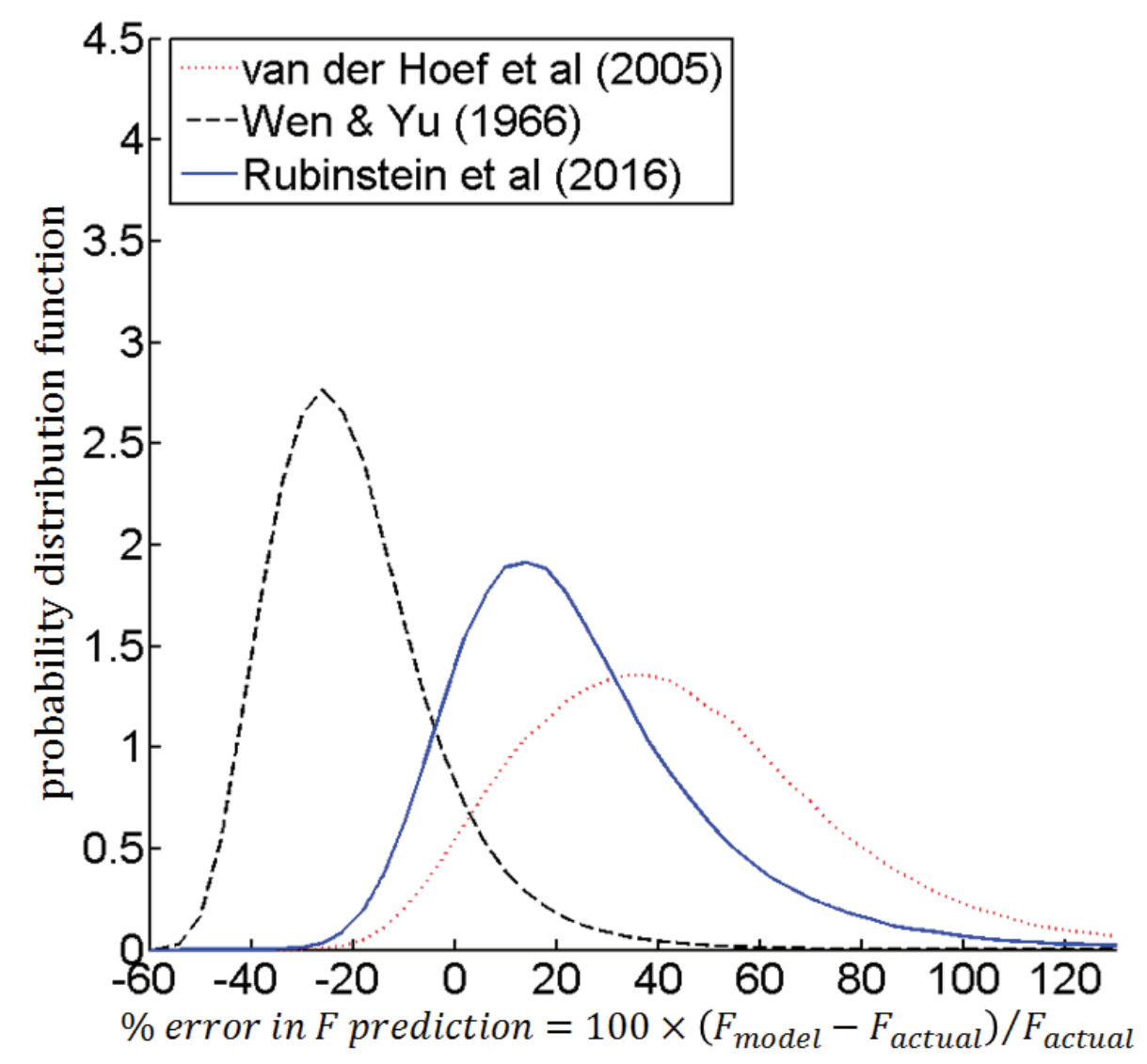

FIGURE 4. The probability distribution functions (PDF) are plotted for the percent error in the drag prediction for three homogeneous drag models: van der Hoef et al. (2005), Wen \& Yu (1966), and Rubinstein et al. (2016). This error analysis is applied to fluidized bed systems with the following set of parameters: $\rho_{p} / \rho_{f}=4,10,100,300,1500$, and 3000 and $\langle\phi\rangle=0.1,0.15,0.2,0.25$, and 0.3 . The drag results are computed using $\Delta_{f} / d_{p}=3$.

\subsection{Error analysis of homogeneous drag models}

As discussed in section 1, prior drag models have been derived using random, homogeneous distributions of particles. However, in practice, these drag models are applied to fluidized bed systems with inhomogeneous microstructures. In order to conduct an analysis of the accuracy of these prior drag models, the filtered $\left(\Delta_{f} / d_{p}=3\right)$ fluid-particle drag, $F$, is computed via fullyresolved simulations in fluidized beds with $\rho_{p} / \rho_{f}=4,10,100,300,1500$, and 3000 and $\langle\phi\rangle=$ $0.1,0.15,0.2,0.25$, and 0.3 , and these LBM drag values are compared with the drag that is predicted by the following constitutive models: van der Hoef et al. (2005), Wen \& Yu (1966), and Rubinstein et al. (2016). In doing so, the error in the model predictions is computed, and the bias and spread in these predictions models are determined. These results are depicted in figure 4 , in which the probability distribution function (PDF) for each model's percent error in the drag prediction is plotted.

For a given drag prediction error PDF curve, the model bias is computed from both the percent error at which the PDF is at a maximum and from the average percent error over the entire distribution. Since the error distributions are slightly skewed towards larger positive errors, the average error is higher than the percent error at the PDF maximum for all of the drag models. The spread in the percent error is computed as half the distance between the two percent error values at which the PDF is at half its maximum. The percent error at the PDF maximum, average error and spread in the errors are summarized in table 1.

As expected, based on the results of figure 4 and table 1, the van der Hoef et al. (2005) 


$\begin{array}{cccc}\text { Drag model } & \begin{array}{c}\text { \% Error at } \\ \text { PDF maximum }\end{array} & \begin{array}{c}\text { Average } \\ \% \text { error }\end{array} & \begin{array}{c}\text { Spread in } \\ \% \text { error }\end{array} \\ \text { van der Hoef } \text { et al. }(2005) & 34.0 \% & 42.8 \% & 34.2 \% \\ \text { Wen \& Yu (1966) } & -26.0 \% & -19.8 \% & 16.5 \% \\ \text { Rubinstein } \text { et al. }(2016) & 14.0 \% & 24.0 \% & 22.8 \%\end{array}$

TABLE 1. The percent error at the maximum of the probability distribution functions, the average percent error, and spread in the distribution of the percent errors of the homogeneous drag models are presented, when these models are applied, using $\Delta_{f} / d_{p}=3$, to fluidized bed systems with the following set of parameters: $\rho_{p} / \rho_{f}=4,10,100,300,1500$, and 3000 and $\langle\phi\rangle=0.1,0.15,0.2,0.25$, and 0.3 .

model, which is a high St limit drag relation, is biased towards overpredicting the drag in finite-St fluidized beds. Analogously, the Wen \& Yu (1966) model, which is a low St limit drag relation, is biased towards underpredicting the drag in finite-St fluidized beds. The Rubinstein et al. (2016) drag model, which accounts for the effect of $S t$, performs better than the two extremes based on the percent errors at the PDF maximum, and better than the van der Hoef et al. (2005) model based on the average percent error. Still, on average, there is a significant drag overprediction $(+14 \%$ error at the PDF maximum and $+24 \%$ average error). This significant overprediction is due to the fact that the Rubinstein et al. (2016) model is unable to capture the effects of inhomogeneities, which, as we found in section 3.1, result in a drag reduction. Overall, by analyzing the prediction errors of the homogeneous drag models, the need for a new drag model that is able to account for the effects of inhomogeneities is clearly demonstrated.

\subsection{Effect of filter size on the behavior of fluidized beds relative to the high and low St limits}

In our prior study focusing on the effect of St (Rubinstein et al. 2016), we found that we could simplify our analysis of the fluid-particle interaction force in a fluidized bed by focusing on the two extreme limits of behavior: high and low St limit beds. In order to connect our findings at these two limits to the behavior of a particular fluidized bed, we developed the parameter, $\alpha$, which quantifies the relative magnitude of the drag in a fluidized bed compared to the high and low St limits:

$$
\alpha=\frac{F_{\text {fluidized }}-F_{\text {low } t, \text { inhom }}}{F_{\text {highSt,inhom }}-F_{\text {low } S t, \text { inhom }}},
$$

where $F_{\text {fluidized }}$ is the dimensionless drag of the fluidized system. In equation 3.2, $F_{\text {high }}$ t, inhom and $F_{\text {low St, inhom }}$ are the drag values computed from the high and low $S t$ limit bed simulations, respectively, which utilize the inhomogeneous configuration of particles that is taken directly from the fluidized system. In our prior work (Rubinstein et al. 2016), we computed $\alpha$ over the full domain size $\left(\Delta_{b}=\Delta_{f}=n_{x}\right)$. In doing so, we found that the results collapse onto a single curve for $\alpha$ as a function of the modified Stokes number, $\tilde{S t}$ (defined in equation 2.5). The domainaveraged function for $\alpha(\tilde{S t})$ is given in equation 2.4. In the present study, we extended this analysis to filter sizes smaller than the domain size, as demonstrated in figure 5. In our current analysis, $\Delta_{b} / d_{p}=1$, and $\alpha$ is computed with $\Delta_{f} / d_{p}=3$ and 5, using fluidized bed simulations with $\rho_{p} / \rho_{f}=4,10,100,300,1500$, and 3000 and $\langle\phi\rangle=0.1,0.15,0.2,0.25$, and 0.3 . In terms of filtered quantities, the modified Stokes number, $\tilde{S t}$, is defined as:

$$
\tilde{S t}=\frac{\rho_{p}\left|\tilde{\boldsymbol{u}}_{s l i p}\right| d_{p}}{18 \mu_{f}(1-\bar{\phi})} .
$$

$\tilde{S t}$ is the filtered version of the modified Stokes number, $S t_{m o d}$, defined in equation 2.5. 


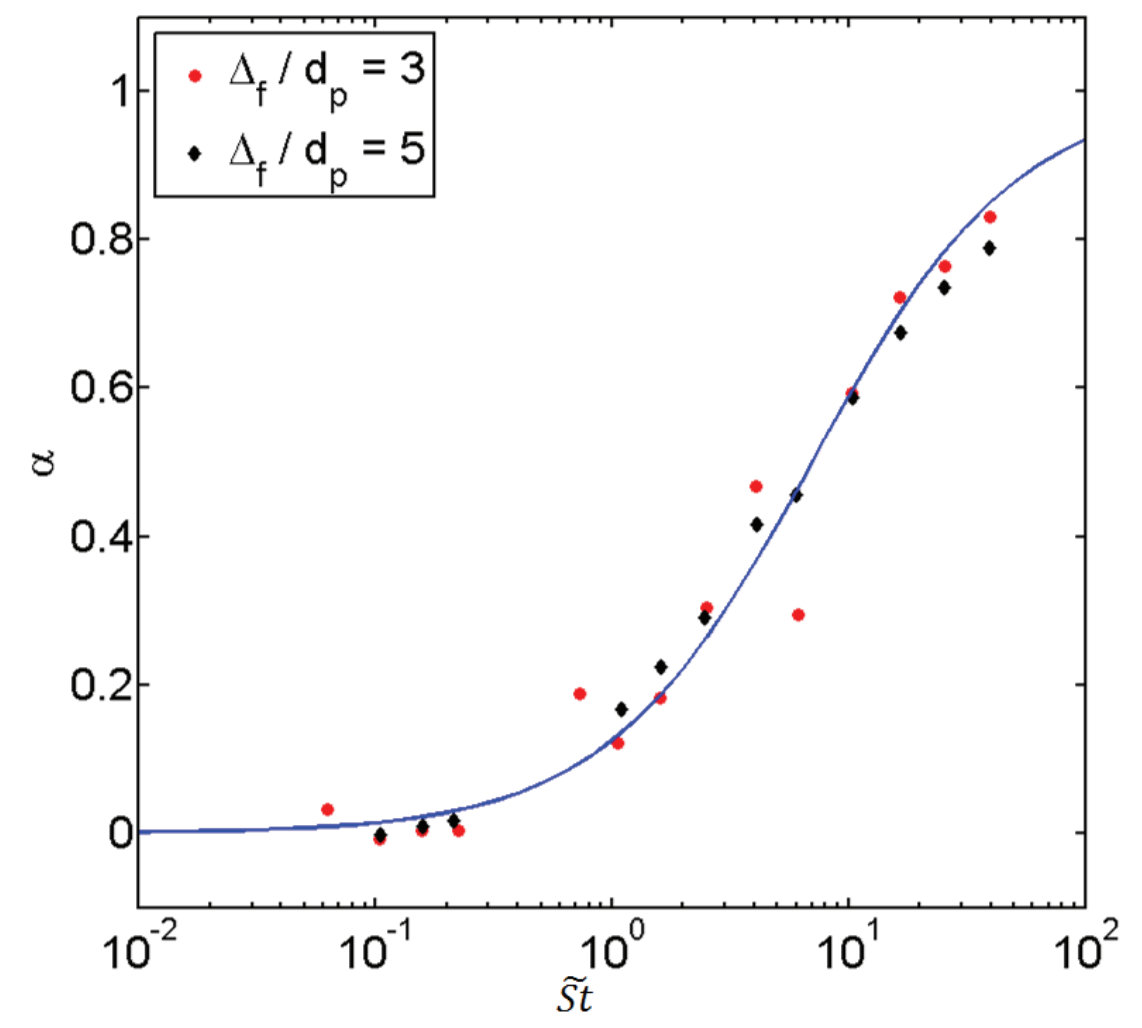

FIGURE 5. The parameter quantifying the behavior of a fluidized bed relative to the high and low $S t$ limits, $\alpha$, is plotted as a function of the modified Stokes number, $\tilde{S t}$, for different filter sizes: $\Delta_{f} / d_{p}=3$ and 5, with $\Delta_{b} / d_{p}=1$. These measurements are taken from simulations of fluidized beds with the following set of parameters: $\rho_{p} / \rho_{f}=4,10,100,300,1500$, and 3000 and $\langle\phi\rangle=0.1,0.15,0.2,0.25$, and 0.3 . The results for $\alpha$ as a function of $\tilde{S t}$ fall onto a single curve, which is given by: $\alpha(\tilde{S t})=\frac{1}{2}\left(1+\frac{\tilde{S} t-7}{\tilde{S} t+7}\right)$.

From figure 5, we see that the results for $\alpha$ as a function of $\tilde{S t}$ computed using different filter sizes all fall on a single curve, which has the following functional form:

$$
\alpha(\tilde{S t})=\frac{1}{2}\left(1+\frac{\tilde{S} t-7}{\tilde{S} t+7}\right) .
$$

Equation 3.4 is nearly identical to equation 2.4, which was derived for our prior domain-averaged analysis (Rubinstein et al. 2016), with the small discrepancy in the coefficient that defines the low-to-high $S t$ limit transition point (7 versus 10) due to a limited sensitivity in the results to the choice of $\Delta_{b}$. The fact that all of the results for $\alpha$ as a function of $\tilde{S t}$ fall onto a single curve signifies that the relationship between $\alpha$ and $\tilde{S t}$ persists even as $\Delta_{f}$ varies, which provides us with a powerful relationship between the fluid-particle drag in a fluidized bed and that in the two limiting cases (high and low St limits). Using this relationship between $\alpha$ and $\tilde{S t}$ at different length scales, our approach for developing a new drag model that accounts for the effects of inhomogeneities is greatly simplified by our ability to focus on the high and low St limit beds, and then interpolate the fluidized bed drag value using the $\alpha(\tilde{S t})$ parameter, rather than having to separately study the effect of inhomogeneities on every different type of fluidized bed.

\subsection{Quantifying the effect of inhomogeneities}

In the low $R e$ regime, where the effects of fluctuations in the particle velocity on the drag force are negligible, changes in the fluid-particle drag of a fluidized bed relative to the random, homogeneous case are due to the extent of inhomogeneities in the distribution of particles. The 
key challenge in developing a new drag model, which is able to accurately describe the behavior of fluidized beds with a wide range of inhomogeneous structures, is therefore to identify how to properly quantify the extent of inhomogeneities. In the current study, three different measures are proposed to quantify the extent of inhomogeneities: the components of the gradient in the particle volume fraction that are perpendicular to the slip velocity, $\nabla \bar{\phi}_{\perp}$, the scalar variance of the particle volume fraction, $\overline{\left(\phi^{\prime}\right)^{2}}$, and the drift flux, $\bar{\phi} \tilde{\boldsymbol{v}}_{d r i f t}$. For the purposes of developing a drag model, the two vector quantities, $\nabla \bar{\phi}_{\perp}$ and $\bar{\phi} \tilde{\boldsymbol{v}}_{\text {drift }}$, are converted into scalars, as discussed in sections 3.4.1 and 3.4.3, respectively.

As summarized in sections 1 and 3.1, $\nabla \bar{\phi}$ has previously been shown by Zhou et al. (2014) and Li et al. (2016) to be a significant measure for determining changes in the drag due to changes in the extents of inhomogeneities, with $\nabla \bar{\phi}_{\perp}$, in particular, directly correlated with drag reduction. The two other measures for the extent of inhomogeneities, $\overline{\left(\phi^{\prime}\right)^{2}}$ and $\bar{\phi} \tilde{\boldsymbol{v}}_{\text {drift }}$, provide additional insight into the effect of inhomogeneities due to their ability to capture sub-grid-scale structures. The scalar variance of the particle volume fraction, $\overline{\left(\phi^{\prime}\right)^{2}}$, is defined as:

$$
\overline{\left(\phi^{\prime}\right)^{2}}=\overline{\phi^{2}}-\bar{\phi}^{2} \text {. }
$$

This form of $\overline{\left(\phi^{\prime}\right)^{2}}$ was previously employed in the context of turbulent combustion modeling by Jiménez et al. (2001), and later used in the context of multi-phase flow modeling by Ozel et al. (2013). The drift flux, $\bar{\phi} \tilde{\boldsymbol{v}}_{\text {drift }}$, measures the correlation between $\boldsymbol{u}_{\text {slip }}$ and $\phi$, and is defined as:

$$
\bar{\phi} \tilde{\boldsymbol{v}}_{\text {drift }}=\overline{\boldsymbol{u}_{\text {slip }} \phi}-\tilde{\boldsymbol{u}}_{\text {slip }} \bar{\phi} .
$$

This drift flux measure was derived concurrently by Simonin and co-workers (Ozel et al. 2010; Parmentier et al. 2012; Ozel et al. 2013) and in the work of Fox (2014). In the next three sections, the effect of each of these three quantities on the drag is further analyzed.

In the current study, filter sizes of 3 and $5 d_{p}$ are employed in order to investigate the effect of inhomogeneities on the drag. Due to the multi-scale hierarachy of the complex structures that develop in a fluidized system, the drag derived in a micro-scale LBM simulation still cannot be directly applied to an industrial-scale TFM or CFD-DEM simulation, which, due to computational restrictions, must have a grid size on the order of $100 d_{p}$. Instead, the goal of this study is to develop a micro-scale drag model that can be applied as a constitutive closure to TFM and CFD-DEM simulations at the meso-scale, where the grid size is $\sim 5 d_{p}$. For those needs, the range of filter sizes that are currently studied are most appropriate. In order to properly account for the effects of the meso-scale structures when simulating industrial-scale fluidized beds, the grid size in the TFM and CFD-DEM simulations needs to be significantly coarsened using a filtering technique, in which a filtered drag relation is derived using meso-scale simulations (Igci \& Sundaresan 2011; Ozel et al. 2013).

\subsubsection{Gradient in the particle volume fraction}

Drawing upon the insights determined by Zhou et al. (2014) and Li et al. (2016), we sought to study the effect of gradients in the particle volume fraction on the fluid-particle drag force. Since, in section 3.1, we found that the dominant effect of inhomogeneities is to produce a reduction in drag, our focus in this study is on $\nabla \bar{\phi}_{\perp}$. In particular, we utilized the following non-dimensional, scalar measure: $\Delta_{f}\left|\nabla \bar{\phi}_{\perp}\right|$. Since this gradient measure is based solely on the configuration of particles, we are able to, based on the conclusions of section 3.3, study the fluidized bed particle configurations using the high and low St limit simulations, and then interpolate these results to the behavior of fluidized beds using $\alpha(\tilde{S t})$, as defined in equation 3.4.

In figure 6 , the dimensionless drag force, $F$, is plotted as a function of $\Delta_{f}\left|\nabla \bar{\phi}_{\perp}\right|$ for the (a) 


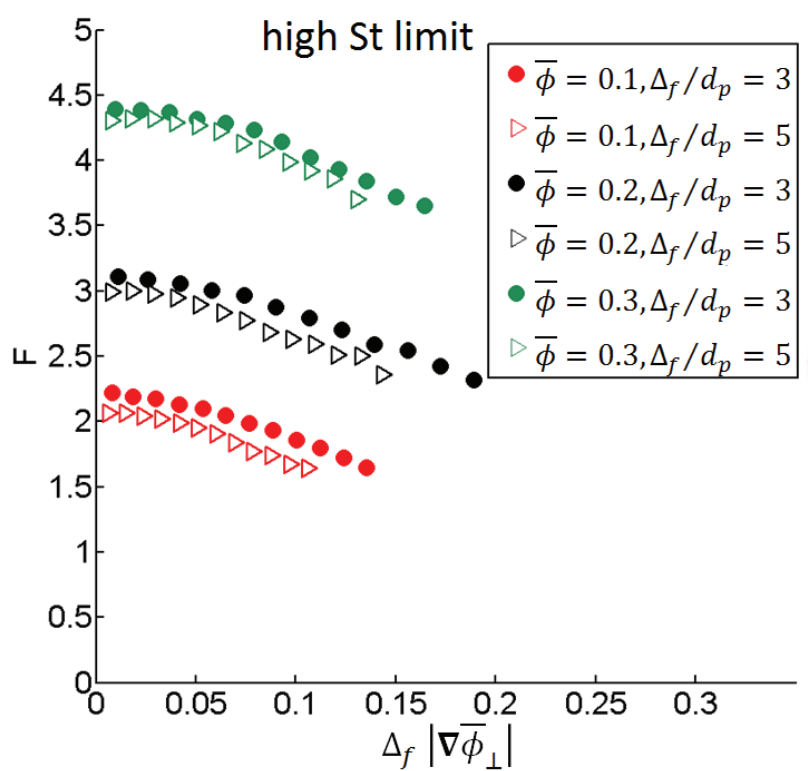

(a)

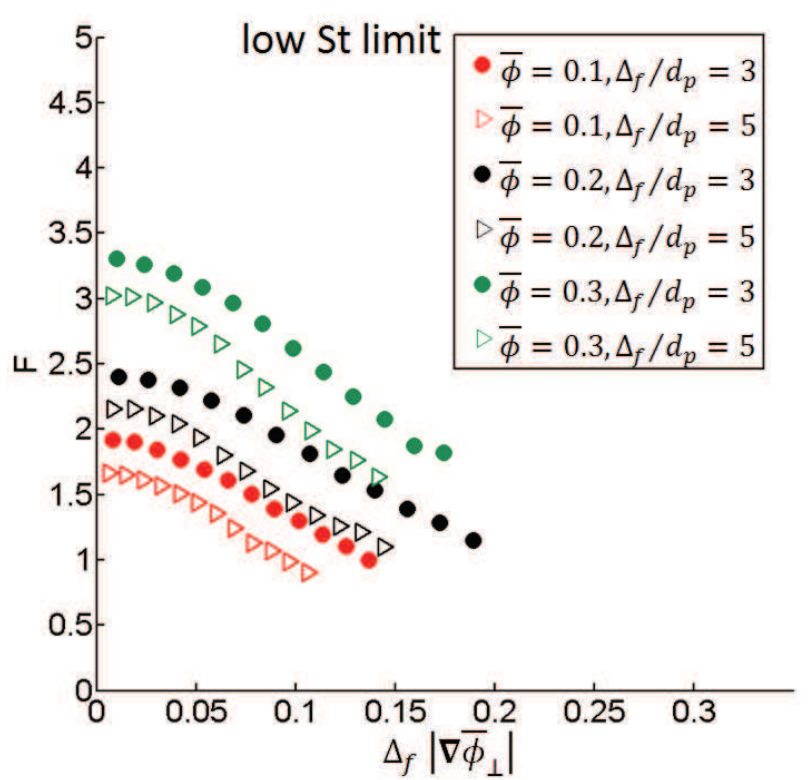

(b)

FIGURE 6. The dimensionless drag force, $F$, is plotted as a function of the dimensionless magnitude of the gradient in particle volume fraction that is perpendicular to the slip velocity, $\Delta_{f}\left|\nabla \bar{\phi}_{\perp}\right|$, for the (a) high $S t$ limit and (b) low St limit. For both of these cases, the results are shown for $\bar{\phi}=0.1,0.2$, and 0.3 and $\Delta_{f} / d_{p}=3$ and 5. These results are compiled from particle configurations taken from fluidized beds with the following set of parameters: $\rho_{p} / \rho_{f}=4,10,100,300,1500$, and 3000 and $\langle\phi\rangle=0.1,0.15,0.2,0.25$, and 0.3 .

high and (b) low St limits. These results are compiled from particle configurations taken from fluidized beds with the following set of parameters: $\rho_{p} / \rho_{f}=4,10,100,300,1500$, and 3000 and $\langle\phi\rangle=0.1,0.15,0.2,0.25$, and 0.3 . For the analysis shown in figure $6, F$ is computed using a range of filter sizes $\left(\Delta_{f} / d_{p}=3\right.$ and 5 ) and bin-averaged with both $\bar{\phi}$ (using a bin width of 0.01 ) and $\Delta_{f}\left|\nabla \bar{\phi}_{\perp}\right|$. In order to demonstrate the effect of $\Delta_{f}\left|\nabla \bar{\phi}_{\perp}\right|$ on $F$, the results are shown for the following $\bar{\phi}$-bins: $\bar{\phi}=0.1,0.2$, and 0.3 . From figures $6 \mathrm{a}$ and $\mathrm{b}$, we observe that, as expected, there is a reduction in drag as $\Delta_{f}\left|\nabla \bar{\phi}_{\perp}\right|$ increases for both the high and low St limits. This trend holds even as $\bar{\phi}$ and $\Delta_{f}$ are both varied.

In order to develop a new $\Delta_{f}\left|\nabla \bar{\phi}_{\perp}\right|$-dependent drag model that can be applied to larger-scale simulations over a range of length scales, it is essential for the trends in the drag force to persist as the filter size is varied. However, from figures $6 a$ and $b$, it is clear that the results for $F$ as a function of $\Delta_{f}\left|\nabla \bar{\phi}_{\perp}\right|$ do not lie on a single curve as $\Delta_{f}$ is varied for both the high and low $S t$ limits. This discrepancy in the trends is especially pronounced in the low $S t$ limit case. Instead, it appears that as $\Delta_{f}$ increases, there is a clear reduction in drag for the same value of $\bar{\phi}$ and $\Delta_{f}\left|\nabla \bar{\phi}_{\perp}\right|$. Choosing the characteristic length scale for the gradient in the volume fraction to be $d_{p}$ instead of $\Delta_{f}$ does not result in a better collapse of the results (not shown in figure 6). Since the $\Delta_{f}\left|\nabla \bar{\phi}_{\perp}\right|$ measure is only capable of detecting inhomogeneities at the scale of the filter size or larger, it appears that at larger filter sizes, the inability of this measure to capture key sub-grid structures, which prior larger-scale filtering studies (Igci \& Sundaresan 2011; Schneiderbauer \& Pirker 2014) have found to have the most significant effect on the drag, prevents it from being a useful method for quantifying the extent of inhomogeneities.

\subsubsection{Scalar variance of the particle volume fraction}

The scalar variance of the particle volume fraction, $\overline{\left(\phi^{\prime}\right)^{2}}$, provides a measure for the extent of fluctuations in the volume fraction at the sub-grid scale. Using $\overline{\left(\phi^{\prime}\right)^{2}}$, as defined in equation 3.5 , 


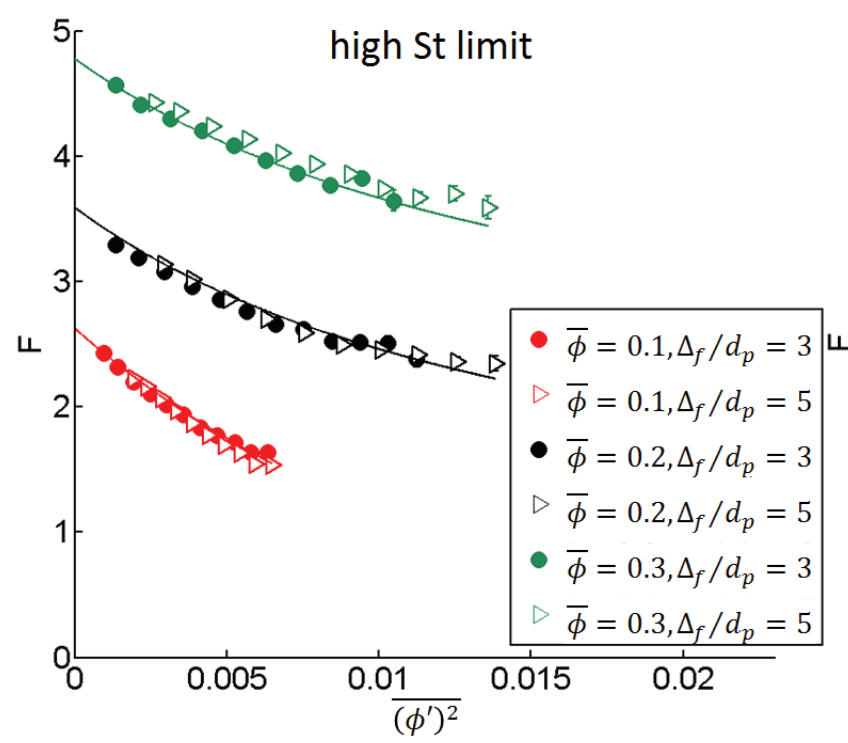

(a)

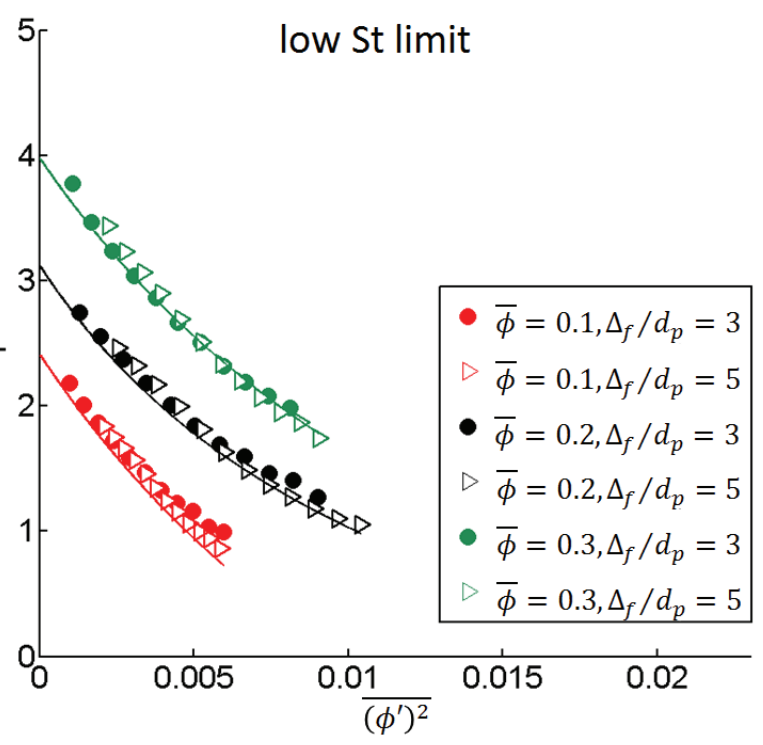

(b)

FIGURE 7. The dimensionless drag force, $F$, is plotted as a function of the scalar variance in particle volume fraction, $\overline{\left(\phi^{\prime}\right)^{2}}$, for the (a) high $S t$ limit and (b) low $S t$ limit. For both of these cases, the results are shown for $\bar{\phi}=0.1,0.2$, and 0.3 and $\Delta_{f} / d_{p}=3$ and 5. These results are compiled from particle configurations taken from fluidized beds with the following set of parameters: $\rho_{p} / \rho_{f}=4,10,100,300,1500$, and 3000 and $\langle\phi\rangle=0.1,0.15,0.2,0.25$, and 0.3 . The curves, derived from the new drag model (equations 3.7 and 3.8) that best fits this data, are shown in these figures.

we sought to model the reduction in drag due to the effects of inhomogeneities. As was the case for the $\Delta_{f}\left|\nabla \bar{\phi}_{\perp}\right|$ measure in section 3.4.1, since $\overline{\left(\phi^{\prime}\right)^{2}}$ is solely based on the configuration of particles, we are able to study the fluidized bed particle configurations using the high and low $S t$ limit simulations, and then interpolate these results to the behavior of fluidized beds using $\alpha(\tilde{S t})$ (equation 3.4).

In figure 7 , the dimensionless drag force, $F$, is plotted as a function of $\overline{\left(\phi^{\prime}\right)^{2}}$ for the (a) high and (b) low St limits. $F$ is computed using a range of filter sizes $\left(\Delta_{f} / d_{p}=3\right.$ and 5) and binaveraged with both $\bar{\phi}$ and $\overline{\left(\phi^{\prime}\right)^{2}}$. These results are compiled from particle configurations taken from fluidized beds with the following set of parameters: $\rho_{p} / \rho_{f}=4,10,100,300,1500$, and 3000 and $\langle\phi\rangle=0.1,0.15,0.2,0.25$, and 0.3 . In order to demonstrate the effect of $\overline{\left(\phi^{\prime}\right)^{2}}$ on $F$, the results are shown for the following $\bar{\phi}$-bins: $\bar{\phi}=0.1,0.2$, and 0.3 . From figures $7 \mathrm{a}$ and $\mathrm{b}$, we observe that, over a range of $\bar{\phi}$ and $\Delta_{f}$ values, there is a strong reduction in drag as $\overline{\left(\phi^{\prime}\right)^{2}}$ increases for both the high and low St limits. In fact, in low St limit systems at $\bar{\phi}=0.1$, there is a $54 \%$ reduction in the $\overline{\left(\phi^{\prime}\right)^{2}}$-bin-averaged dimensionless drag force, $F$, over the range of observed $\overline{\left(\phi^{\prime}\right)^{2}}$ values, computed using $\Delta_{f}=3 d_{p}$. This strong reduction in $F$ is completely undetected by prior drag relations that do not account for the extent of inhomogeneities.

In contrast to the observations made from figure 6 for the $\Delta_{f}\left|\nabla \bar{\phi}_{\perp}\right|$ measure, in figure 7 , we clearly see that, for a particular value of $\bar{\phi}$, the results for $F$ as a function of $\overline{\left(\phi^{\prime}\right)^{2}}$ lie on a single curve as $\Delta_{f}$ is varied. Such a result suggests that $\overline{\left(\phi^{\prime}\right)^{2}}$ provides a powerful measure for the extent of inhomogeneities over a range of length scales. Even at larger filter sizes, $\overline{\left(\phi^{\prime}\right)^{2}}$ is still able to capture the effects of key sub-grid-scale structures. Having established the utility of the $\overline{\left(\phi^{\prime}\right)^{2}}$ measure, we then sought to model the drag results at both the high and low St limits. The high $S t$ 
Drag model function

high St limit

$$
\begin{array}{cc}
F_{0}(\bar{\phi}) & 8.54 \frac{\bar{\phi}}{1-\bar{\phi}}+(1-\bar{\phi})^{3}(1+4.11 \sqrt{\bar{\phi}}) \\
a(\bar{\phi}) & 5.70-221\left(\frac{\bar{\phi}}{\phi_{\max }}\right)^{2.5}\left(1-\frac{\bar{\phi}}{\phi_{\max }}\right)^{4} \\
b(\bar{\phi}) & 0.0220-240\left(\frac{\bar{\phi}}{\bar{\phi}_{\max }}\right)^{6}\left(1-\frac{\bar{\phi}}{\phi_{\max }}\right)^{10}
\end{array}
$$

low $S t$ limit

TABLE 2. The $\overline{\left(\phi^{\prime}\right)^{2}}$-dependent drag model functions, $F_{0}, a$, and $b$, are presented for both the high and low St limits, with $\phi_{\max }=0.64$.

and low St limit drag models are written as:

$$
\begin{gathered}
F_{\text {highSt }}\left(\bar{\phi}, \overline{\left(\phi^{\prime}\right)^{2}}\right)=F_{0, \text { high St }}(\bar{\phi})-a_{\text {high } S t}(\bar{\phi}) \frac{\overline{\left(\phi^{\prime}\right)^{2}} / b_{\text {highSt }}(\bar{\phi})}{1+\overline{\left(\phi^{\prime}\right)^{2}} / b_{\text {high } S t}(\bar{\phi})}, \\
F_{\text {low } S t}\left(\bar{\phi}, \overline{\left(\phi^{\prime}\right)^{2}}\right)=F_{0, \text { low } S t}(\bar{\phi})-a_{\text {low } S t}(\bar{\phi}) \frac{\overline{\left(\phi^{\prime}\right)^{2}} / b_{\text {low } S t}(\bar{\phi})}{1+\overline{\left(\phi^{\prime}\right)^{2}} / b_{\text {low } S t}(\bar{\phi})} .
\end{gathered}
$$

In equations 3.7 and 3.8, the model functions $F_{0}, a$, and $b$ are defined in table 2. The functional form of $F_{0}$ in table 2 is inspired by the van der Hoef et al. (2005) drag model in equation 2.2. The functional form of $a$ and $b$ in table 2 is empirically derived from this simulation study.

The solid curves drawn in figures $7 \mathrm{a}$ and $\mathrm{b}$ are based on the drag models in equations 3.7 and 3.8 , respectively. The ability of these high and low $S t$ limit drag relations to accurately fit the drag results over a range of $\bar{\phi}$ and $\overline{\left(\phi^{\prime}\right)^{2}}$ demonstrates the success of our model development.

Using the high and low $S t$ limit drag models, we then interpolate the drag value for a fluidized bed with the following equation:

$$
F\left(\bar{\phi}, \tilde{S t}, \overline{\left(\phi^{\prime}\right)^{2}}\right)=\alpha(\tilde{S t}) F_{\text {highSt }}\left(\bar{\phi}, \overline{\left(\phi^{\prime}\right)^{2}}\right)+(1-\alpha(\tilde{S t})) F_{\text {low } S t}\left(\bar{\phi}, \overline{\left(\phi^{\prime}\right)^{2}}\right)
$$

where $\alpha(\tilde{S t})$ is defined in equation 3.4 and $\tilde{S t}$ is defined in equation 3.3. Using equations 3.73.9, we are able to define a new $\overline{\left(\phi^{\prime}\right)^{2}}$-dependent drag model that accounts for the effects of inhomogeneities over a range of length scales. In applying this drag relation as a closure to larger-scale simulations, where the flow is not resolved down to the $1 d_{p}$ scale, a method for estimating $\overline{\left(\phi^{\prime}\right)^{2}}$ is needed. In order to estimate the value of this quantity, we need to solve an additional transport equation for $\overline{\left(\phi^{\prime}\right)^{2}}$ or employ a scale-similarity approach, as described in section 3.5.1.

\subsubsection{Drift flux}

As an alternative to defining a drag model in terms of $\overline{\left(\phi^{\prime}\right)^{2}}$, we can also define a drag model in which the extent of inhomogeneities is captured through the drift flux (Ozel et al. 2013), as defined in equation 3.6. This quantity is based not only on the configuration of particles, but also on how the flow structures correlate with particle distributions. A normalized measure for the drift flux is given as:

$$
v_{d}=\frac{\left(\bar{\phi} \tilde{\boldsymbol{v}}_{\text {drift }}\right) \cdot \tilde{\boldsymbol{u}}_{\text {slip }}}{\tilde{\boldsymbol{u}}_{\text {slip }} \cdot \tilde{\boldsymbol{u}}_{\text {slip }}}
$$




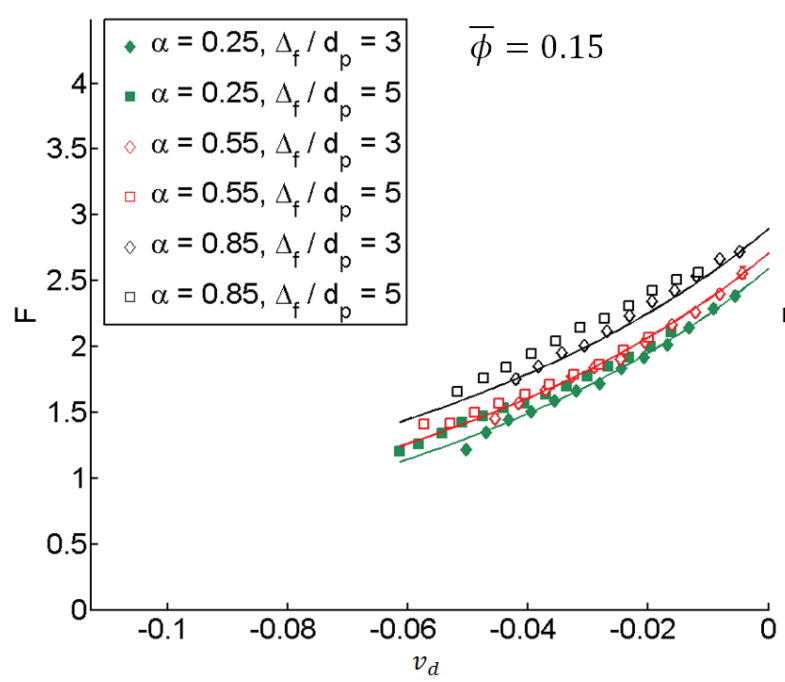

(a)

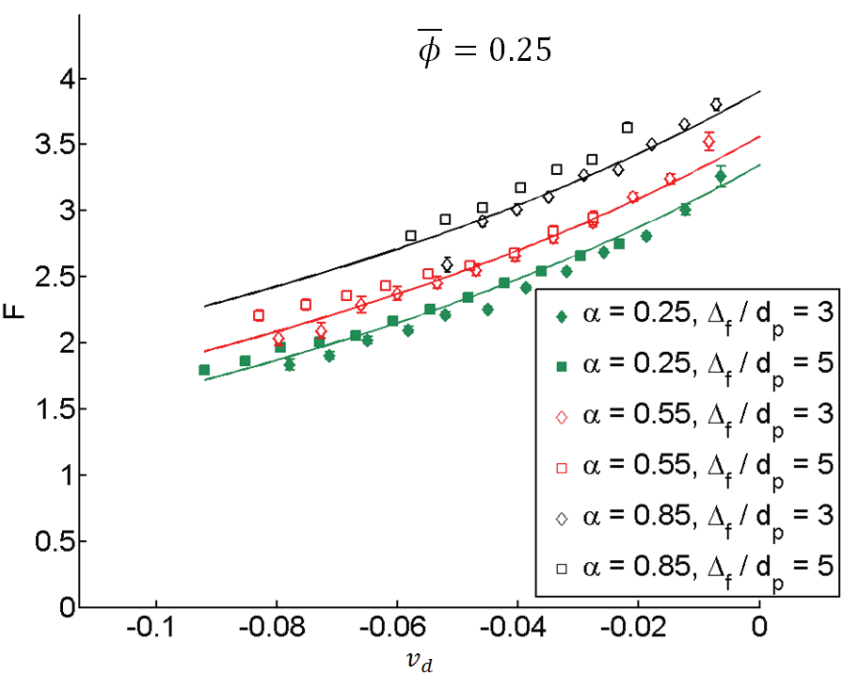

(b)

FIGURE 8. The dimensionless drag force, $F$, is plotted as a function of the dimensionless drift flux, $v_{d}$, for (a) $\bar{\phi}=0.15$ and (b) $\bar{\phi}=0.25$. For both of these cases, the results are shown for $\alpha=0.25,0.55$, and 0.85 and $\Delta_{f} / d_{p}=3$ and 5 . These results are compiled from fluidized bed simulations with the following set of parameters: $\rho_{p} / \rho_{f}=4,10,100,300,1500$, and 3000 and $\langle\phi\rangle=0.1,0.15,0.2,0.25$, and 0.3 . The curves, derived from the new drag model (equation 3.11) that best fits this data, are shown in these figures.

$v_{d}$, which is a scalar quantity, is defined as the normalized component of the drift flux that is in the direction of the slip velocity. Since we found that the magnitude of the drift flux components perpendicular to the slip velocity is negligible $(<1 \%)$ relative to that of the parallel components, we are able to focus solely on the parallel components for the purposes of drag modeling. Since the drift flux is not solely based on the configuration of particles, it is not useful to derive the drag model based on the drift flux only using high and low $S t$ limit simulations. Thus, we are unable to employ the same methodology for developing a $v_{d}$-dependent drag model as we did with the $\overline{\left(\phi^{\prime}\right)^{2}}$-based model in section 3.4.2.

In figure $8, F$ is plotted as a function of $v_{d}$, using the results of fluidized bed simulations with the following set of parameters: $\rho_{p} / \rho_{f}=4,10,100,300,1500$, and 3000 and $\langle\phi\rangle=0.1,0.15$, $0.2,0.25$, and $0.3 . F$ is bin-averaged with $\bar{\phi}, v_{d}$, and $\alpha$ for the following filter sizes: $\Delta_{f} / d_{p}=3$ and 5. The value of $\alpha$, as defined in equation 3.2, is computed at each filter cell by comparing the drag results in a particular fluidized bed with those in its inhomogeneous high and low $S t$ limit counterparts. In figures $8 \mathrm{a}$ and $\mathrm{b}$, the results for the $\bar{\phi}=0.15$ and $\bar{\phi}=0.25$ bins are shown, respectively. In order to fully demonstrate the effect of $v_{d}$ on $F$, the results in figure 8 are shown for the following $\alpha$-bins: $\alpha=0.25,0.55$, and 0.85 , with $\alpha$-bin widths of 0.1 . From figures 8 a and $\mathrm{b}$, we observe that, over a range of $\bar{\phi}, \Delta_{f}$, and $\alpha$ values, there is a reduction in drag as $v_{d}$ becomes more negative. In fact, at $\bar{\phi}=0.15$ and $\alpha=0.25$, there is a $49 \%$ reduction in the $v_{d}$-bin-averaged dimensionless drag force, $F$, over the range of observed drift flux values, computed using $\Delta_{f}=$ $3 d_{p}$. Such a pronounced reduction in $F$ cannot be detected by the Rubinstein et al. (2016) model (equation 2.1). It is important to point out that the dimensionless drift flux is primarily negative over the entire fluidized bed system. Since the drift flux is a measure of the correlation between $\boldsymbol{u}_{\text {slip }}$ and $\phi$, this correlation is typically negative due to the fact that higher values of $\phi$ usually result in lower magnitudes of $\boldsymbol{u}_{\text {slip }}$.

In figure 8, for a particular pair of $\bar{\phi}$ and $\alpha$ values, the results for $F$ as a function of $v_{d}$ fall on a single curve as $\Delta_{f}$ is varied. Such a result suggests that the drift flux provides a powerful measure for the extent of inhomogeneities over a range of length scales. Since $v_{d}$, like $\overline{\left(\phi^{\prime}\right)^{2}}$, is a sub-grid-scale quantity, this measure is still able to capture the effects of key small-scale 


$$
\begin{array}{cc}
\text { Drag model function } & \text { Functional value } \\
F_{0, \text { high } S t}(\bar{\phi}) & 8.41 \frac{\bar{\phi}}{1-\bar{\phi}}+(1-\bar{\phi})^{3}(1+3.45 \sqrt{\bar{\phi}}) \\
F_{0, \text { low } S t}(\bar{\phi}) & 6.57 \frac{\bar{\phi}}{1-\bar{\phi}}+(1-\bar{\phi})^{3}(1+3.39 \sqrt{\bar{\phi}}) \\
a(\bar{\phi}) & 10.95-24.82\left(\frac{\bar{\phi}}{\phi_{\max }}\right)^{0.5}\left(1-\frac{\bar{\phi}}{\phi_{\max }}\right)^{2} \\
b(\bar{\phi}) & -0.093-6460\left(\frac{\bar{\phi}}{\phi_{\max }}\right)^{8}\left(1-\frac{\bar{\phi}}{\phi_{\max }}\right)^{7}
\end{array}
$$

TABLE 3 . The drift flux-dependent drag model functions, $F_{0, h i g h S t}, F_{0, l o w S t}, a$, and $b$, are presented, with $\phi_{\max }=0.64$.

structures, even at larger filter sizes. Thus, we sought to model the drag as a function of the drift flux.

The drift flux-dependent drag model is defined as follows:

$$
F\left(\bar{\phi}, \tilde{S t}, v_{d}\right)=F_{0}(\bar{\phi}, \alpha(\tilde{S t}))-a(\bar{\phi}) \frac{v_{d} / b(\bar{\phi})}{1+v_{d} / b(\bar{\phi})}
$$

where $F_{0}(\bar{\phi})$ is defined as:

$F_{0}(\bar{\phi}, \alpha(\tilde{S t}))=\frac{F_{0, h i g h S t}(\bar{\phi})+F_{0, \text { low } S t}(\bar{\phi})}{2}+\left(\frac{F_{0, h i g h S t}(\bar{\phi})-F_{0, \text { low } S t}(\bar{\phi})}{2}\right) \frac{e^{10(\alpha(\tilde{S t})-0.6)}-1}{e^{10(\alpha(\tilde{S t})-0.6)}+1}$.

The functional form in equation 3.12 is based on the functional form for $\alpha(\tilde{S t})$, as given in equation 3.4, with $F_{0} \rightarrow F_{0, \text { high St }}$ and $F_{0} \rightarrow F_{0, \text { low } S t}$ at the high and low $S t$ limits, respectively. In equations 3.11 and 3.12 , the model functions $F_{0, h i g h S t}(\bar{\phi}), F_{0, l o w S t}(\bar{\phi}), a(\bar{\phi})$, and $b(\bar{\phi})$ are defined in table 3. The functional form of $F_{0, \text { high } S t}$ and $F_{0, \text { low } S t}$ in table 3 is inspired by the van der Hoef et al. (2005) drag model in equation 2.2. The functional form of $a$ and $b$ in table 3 is empirically derived from this simulation study.

The solid curves drawn in figures $8 \mathrm{a}$ and $\mathrm{b}$ are based on the drag model in equation 3.11. The ability of this drag relation to accurately fit the drag results over a range of $\bar{\phi}, v_{d}$, and $\alpha(\tilde{S t})$ demonstrates the success of our model development. Using equations 3.11 and 3.12 and the model functions of table 3 , we are able to define a new $v_{d}$-dependent drag model that accounts for the effects of inhomogeneities over a range of length scales. As was the case for the $\overline{\left(\phi^{\prime}\right)^{2}}$ dependent drag model, in applying this drag relation as a closure to larger-scale simulations, a method for estimating $v_{d}$ is needed since the flow is not resolved down to the $1 d_{p}$ scale. In order to estimate $v_{d}$, we need to solve an additional transport equation for $v_{d}$ or employ a scale-similarity approach, as described in section 3.5.2.

\subsection{Scale-similar modeling of sub-grid-scale quantities}

The new drag models that are proposed in section 3.4 quantify the extent of inhomogeneities through one of two sub-grid-scale measures: $\overline{\left(\phi^{\prime}\right)^{2}}$ or $v_{d}$. In LBM simulations, the flow is fully resolved at the $1 d_{p}$ length scale of the base grid cell, and so these quantities can be directly computed using flow information that is available at the sub-filter scale. However, when these drag relations are applied as constitutive closures to larger-scale simulations, where the flow is not resolved at the $1 d_{p}$ length scale and where $\Delta_{f}$ and $\Delta_{b}$ are both taken to be equal to the 
fluid grid size, the sub-grid-scale quantities cannot be directly computed. Thus, a method for estimating these sub-grid quantities is needed.

In this study, we employ a scale-similar approach to model the sub-grid-scale quantities. This scale-similar approach was first introduced in modeling eddy viscosity in the context of single phase turbulence by Germano et al. (1991), and was later adapted to drag modeling in largescale multi-phase flow modeling by Parmentier et al. (2012) and Ozel et al. (2013). Such an approach has not previously been used in the context of developing constitutive models for the fluid-particle drag force using smaller-scale, fully-resolved simulations.

In the scale-similar approach, the key assumption made is that the type of inhomogeneous structures that occur at a particular length scale can be predicted based on the type of structures that occur at a second length scale. Thus, using scale-similarity, information available at or above the grid length scale can be used to approximate the sub-grid quantities. In order to utilize the scale-similar assumption, a model of the following form needs to be proposed for the sub-filter quantities:

$$
q=\kappa f\left(\bar{\phi} / \phi_{\max }\right) g\left(\Delta_{f} / d_{p}\right)
$$

where $q$ is a sub-grid-scale measure for the extent of inhomogeneities. $f\left(\bar{\phi} / \phi_{\max }\right)$ and $g\left(\Delta_{f} / d_{p}\right)$ define the functional dependence of the sub-grid quantity on the particle concentration and mesh, respectively, and are applicable to fluidized beds with a wide range of system properties. In equation 3.13, $\kappa$ is a scalar quantity that adjusts based on the extent of inhomogeneities in a particular system. While $\kappa$ will vary from system to system, for a particular system, the value of $\kappa$ will persist over a range of length scales (filter sizes), based on the scale-similarity assumption. The functional form in equation 3.13 is based on the work of Parmentier et al. (2012) and Ozel et al. (2013).

In order to proceed with this scale-similar modeling approach, the first step is develop accurate expressions for $f$ and $g$. Our development of these models are described in sections 3.5.1 and 3.5.2 for $\overline{\left(\phi^{\prime}\right)^{2}}$ and $v_{d}$, respectively. Once models for $f$ and $g$ have been developed, in order to estimate the sub-grid quantity in equation 3.13, we still need a method to approximate the value of $\kappa$. In order to take advantage of the scale-similarity assumption, $\kappa$ is computed through a technique in which the flow structures are analyzed using a second, larger filter size, known as the test filter, $\Delta_{f, t e s t}$. Since the flow is resolved at length scales below $\Delta_{f, t e s t}$, the use of this test filter approach allows us to compute the extent of sub-filter-scale inhomogeneities at the $\Delta_{f, t e s t}$ length scale. Since the value of $\kappa$ holds at both $\Delta_{f}$ and $\Delta_{f, t e s t}$, the value of $\kappa$ can be determined for all of the fluidized bed systems. Having determined $f, g$, and $\kappa$, the sub-grid-scale measure for the extent of inhomogeneities can then be approximated. While some of the mechanics of this test filter approach are presented in section 3.5.1 for the modeling of $\overline{\left(\phi^{\prime}\right)^{2}}$, further details can be found in the work of Ozel et al. (2013).

\subsubsection{Scalar variance of particle volume fraction}

Since $\overline{\left(\phi^{\prime}\right)^{2}}$ is a sub-grid-scale measure for the extent of inhomogeneities, we employ a scalesimilar approach to estimate this quantity. In order to utilize this approach, the scale-similar form for $\overline{\left(\phi^{\prime}\right)^{2}}$ is defined as:

$$
\overline{\left(\phi^{\prime}\right)^{2}}=\kappa_{1} f_{1}\left(\bar{\phi} / \phi_{\max }\right) g_{1}\left(\Delta_{f} / d_{p}\right)
$$

which is based on the generic scale-similar formalism in equation 3.13

In order to determine a model for $f_{1}$, the dependence of $\overline{\left(\phi^{\prime}\right)^{2}}$ on the concentration of particles, the value of $\overline{\left(\phi^{\prime}\right)^{2}}$ is evaluated for fluidized bed configurations with a wide range of parameters. 


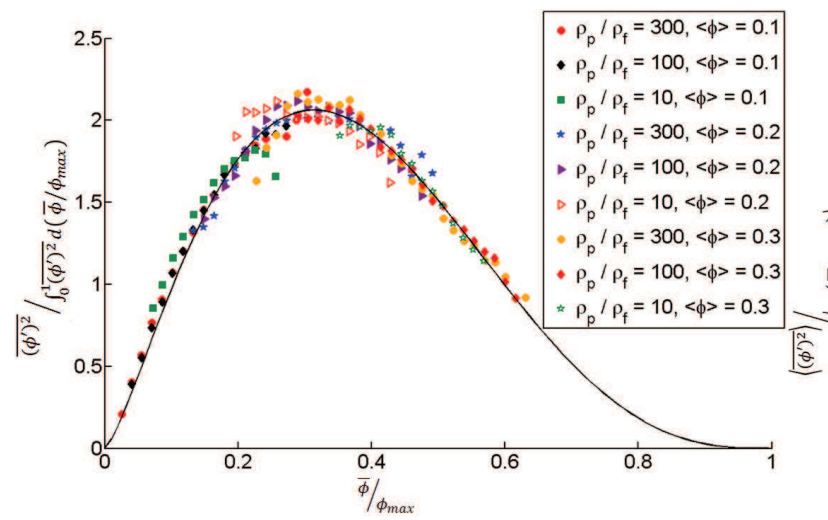

(a)

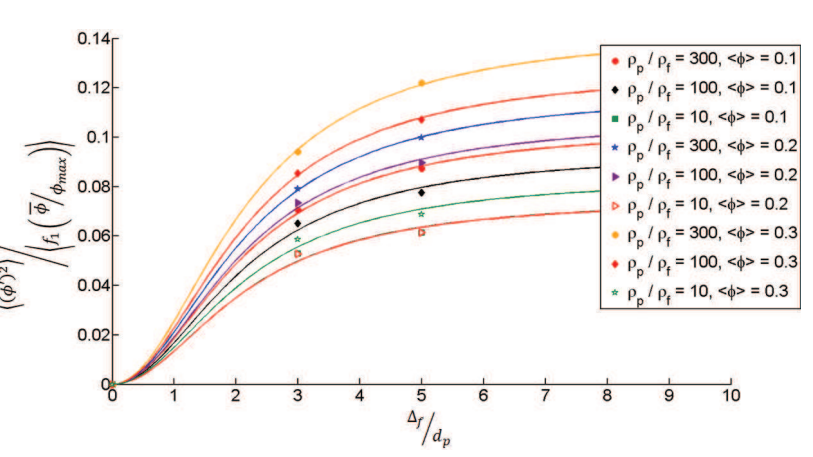

(b)

FIGURE 9. (a) The results for $\overline{\left(\phi^{\prime}\right)^{2}}$, normalized by $\int_{0}^{1} \overline{\left(\phi^{\prime}\right)^{2}} d\left(\bar{\phi} / \phi_{\max }\right)$, are shown as a function of $\bar{\phi} / \phi_{\max }$. These results are computed using $\Delta_{f} / d_{p}=3$. The results from the different particle configurations all fall onto a single curve, which we define as: $f_{1}\left(\bar{\phi} / \phi_{\max }\right)=\left(\bar{\phi} / \phi_{\max }\right)^{1.37}\left(1-\bar{\phi} / \phi_{\max }\right)^{3.00}$. (b) The domain-averaged scalar variance results, $\left\langle\overline{\left(\phi^{\prime}\right)^{2}}\right\rangle$, normalized by $\left\langle f_{1}\left(\bar{\phi} / \phi_{\max }\right)\right\rangle$, are shown as a function of $\Delta_{f} / d_{p}$. The results from the different particle configurations are all modeled using a single function, $\left\langle\kappa_{1}\right\rangle g_{1}\left(\Delta_{f} / d_{p}\right)=\left\langle\kappa_{1}\right\rangle \frac{\left(\Delta_{f} / d_{p}\right)^{2}}{4.58+\left(\Delta_{f} / d_{p}\right)^{2}}$, which provides the functional form for the different fitting curves. The results for both of these figures are compiled from particle configurations taken from fluidized beds with the following set of parameters: $\rho_{p} / \rho_{f}=10,100$, and 300 and $\langle\phi\rangle=0.1,0.2$, and 0.3 .

This analysis is shown in figure $9 \mathrm{a}$, where $f_{1}$ is determined as:

$$
f_{1}\left(\bar{\phi} / \phi_{\max }\right)=\frac{\overline{\left(\phi^{\prime}\right)^{2}}}{\int_{0}^{1} \overline{\left(\phi^{\prime}\right)^{2}} d\left(\bar{\phi} / \phi_{\max }\right)} .
$$

In order to compute the integral in the denominator of equation 3.15, a curve is drawn through the results of $\overline{\left(\phi^{\prime}\right)^{2}}$ as a function of $\bar{\phi} / \phi_{\max }$ from $\bar{\phi} / \phi_{\max }=0$ to $\bar{\phi} / \phi_{\max }=1 . \overline{\left(\phi^{\prime}\right)^{2}}$ is forced to 0 at these two limits, since there is no variance in the volume fraction when no particles are present and when the particles are at a maximum packing fraction. Using a filter size of $\Delta_{f} / d_{p}=3$, the results for $\overline{\left(\phi^{\prime}\right)^{2}} / \int_{0}^{1} \overline{\left(\phi^{\prime}\right)^{2}} d\left(\bar{\phi} / \phi_{\max }\right)$ are then computed as a function of $\bar{\phi} / \phi_{\max }$. These results are compiled from particle configurations taken from fluidized beds with the following set of parameters: $\rho_{p} / \rho_{f}=10,100$, and 300 and $\langle\phi\rangle=0.1,0.2$, and 0.3. From figure 9a, it is clear that the results from these different configurations all fall onto a single curve, with the following functional form:

$$
f_{1}\left(\bar{\phi} / \phi_{\max }\right)=\left(\bar{\phi} / \phi_{\max }\right)^{n_{1}}\left(1-\bar{\phi} / \phi_{\max }\right)^{m_{1}}
$$

where $n_{1}=1.37, m_{1}=3.00$, and $\phi_{\max }=0.64$. The function $f_{1}\left(\bar{\phi} / \phi_{\max }\right)$, defined in equation 3.16 and derived using $\Delta_{f} / d_{p}=3$, is valid over a range of length scales, as it has also been shown to hold at $\Delta_{f} / d_{p}=5$ to within $4 \%$. In addition, while figure 9a provides only a sampling of the fluidized beds that were simulated in the current study, the universal shape of equation 3.16 holds over the full range of $\rho_{p} / \rho_{f}(4-3000)$ and $\langle\phi\rangle(0.1-0.3)$ that were investigated. Furthermore, while the high computational demands associated with properly resolving the flow between particles at very high particle volume fractions prevent us from sampling $\bar{\phi}$ between 0.4 and $\phi_{\max }$ in the current work, the fact that the universal shape holds over the entire sampled $\bar{\phi}$ and that prior multiphase flow studies employing a scale-similarity technique (Ozel et al. 2013) have found a similar concentration dependence suggest that equation 3.16 should hold over the full range of $\bar{\phi}$.

Next, in order to determine a model for $g_{1}$, which defines the mesh-dependence of $\overline{\left(\phi^{\prime}\right)^{2}}$, the 
properties of $\overline{\left(\phi^{\prime}\right)^{2}}$ are studied over a range of filter sizes. This analysis is shown in figure $9 \mathrm{~b}$, where $g_{1}$ is determined as:

$$
g_{1}\left(\Delta_{f} / d_{p}\right)=\frac{\left\langle\overline{\left(\phi^{\prime}\right)^{2}}\right\rangle}{\left\langle\kappa_{1} f_{1}\left(\bar{\phi} / \phi_{\max }\right)\right\rangle},
$$

with $f_{1}$ defined by equation 3.16. Performing the domain-averaging operation in equation 3.17 allows for us to determine how, on average, $\overline{\left(\phi^{\prime}\right)^{2}}$ changes with filter size. In figure $9 \mathrm{~b}$, the results for $\left.\overline{\left\langle\left(\phi^{\prime}\right)^{2}\right.}\right\rangle /\left\langle f_{1}\left(\bar{\phi} / \phi_{\max }\right)\right\rangle$, are shown as a function of $\Delta_{f} / d_{p}$. The results for each different configuration have the same dependence on $\Delta_{f} / d_{p}$ and $g_{1}\left(\Delta_{f} / d_{p}\right)$, and simply vary by the multiplicative constant, $\left\langle\kappa_{1}\right\rangle$, which defines the extent of inhomogeneities for a particular system, averaged over the domain. This functional dependence on $\Delta_{f} / d_{p}$ is found to be:

$$
g_{1}\left(\Delta_{f} / d_{p}\right)=\frac{\left(\Delta_{f} / d_{p}\right)^{2}}{k_{1}+\left(\Delta_{f} / d_{p}\right)^{2}}
$$

where $k_{1}=4.58$. While larger computational domains are necessary in order to fully probe the validity of the quadratic mesh dependence in the smaller filter size regime, the form of equation 3.18 is confirmed in the current study through its ability to effectively pass through all of the data points in figure $9 \mathrm{~b}$ and by the fact that this equation is consistent with the Taylor series expansion of the drift velocity that is performed by Ozel et al. (2013). The value for $k_{1}$ is determined based on a best fit of the simulation data points in figure $9 \mathrm{~b}$. The curves, based on $\left\langle\kappa_{1}\right\rangle g_{1}\left(\Delta_{f} / d_{p}\right)$, are shown, in figure $9 \mathrm{~b}$, to fit the results over a range of system parameters. $\kappa_{1}$ is found to be larger for particle configurations taken from fluidized beds with larger values of $\rho_{p} / \rho_{f}$, due to the fact that these configurations have a larger extent of inhomogeneities. As an example of this trend, for systems with $\langle\phi\rangle=0.3$, the domain-averaged value of $\kappa_{1}$ is 0.084 and 0.143 for the $\rho_{p} / \rho_{f}=10$ and $\rho_{p} / \rho_{f}=300$ configurations, respectively.

Having developed the scale-similar models for $f_{1}\left(\bar{\phi} / \phi_{\max }\right)$ and $g_{1}\left(\Delta_{f} / d_{p}\right), \overline{\left(\phi^{\prime}\right)^{2}}$ can then be estimated by utilizing the test filter approach, as discussed in section 3.5. Quantities that are filtered using the test filter, $\Delta_{f, \text { test }}$, are denoted with ${ }^{\wedge}$. The scalar variance of the filtered solid volume fraction, $\widehat{\widehat{\left(\phi^{\prime}\right)_{*}^{2}}}$, at the test scale is defined as:

$$
\widehat{\widehat{\left(\phi^{\prime}\right)_{*}^{2}}}=\widehat{\overline{\phi^{2}}}-\widehat{\bar{\phi}}^{2}=\kappa_{1} f_{1}\left(\widehat{\bar{\phi}} / \phi_{\max }\right) g_{1}\left(\Delta_{f, t e s t} / d_{p}\right)
$$

while the test-filtered scalar variance, $\widehat{\overline{\left(\phi^{\prime}\right)^{2}}}$, is defined as:

$$
\widehat{\overline{\left(\phi^{\prime}\right)^{2}}}=\overline{\overline{\phi^{2}}-\bar{\phi}^{2}}=\kappa_{1} \overline{f_{1}\left(\bar{\phi} / \phi_{\max }\right) g_{1}\left(\Delta_{f} / d_{p}\right)} \text {. }
$$

From equations 3.19 and 3.20, we are able to solve for $\kappa_{1}$ as:

$$
\kappa_{1}=\frac{\widehat{\bar{\phi}}^{2}-\widehat{\bar{\phi}}^{2}}{f_{1}\left(\widehat{\bar{\phi}} / \phi_{\max }\right) g_{1}\left(\Delta_{f, \text { test }} / d_{p}\right)-\overline{f_{1}\left(\bar{\phi} / \phi_{\max }\right) g_{1}\left(\Delta_{f} / d_{p}\right)}} .
$$

Using this test filter approach, the value of $\kappa_{1}$ can be estimated throughout the fluidized bed system.

The sub-grid-scale measure for the extent of inhomogeneities, $\overline{\left(\phi^{\prime}\right)^{2}}$, can be approximated through scale-similar modeling using equations 3.14, 3.16, 3.18, and 3.21. In doing so, this quantity can be estimated in simulations where the flow is not fully resolved. 


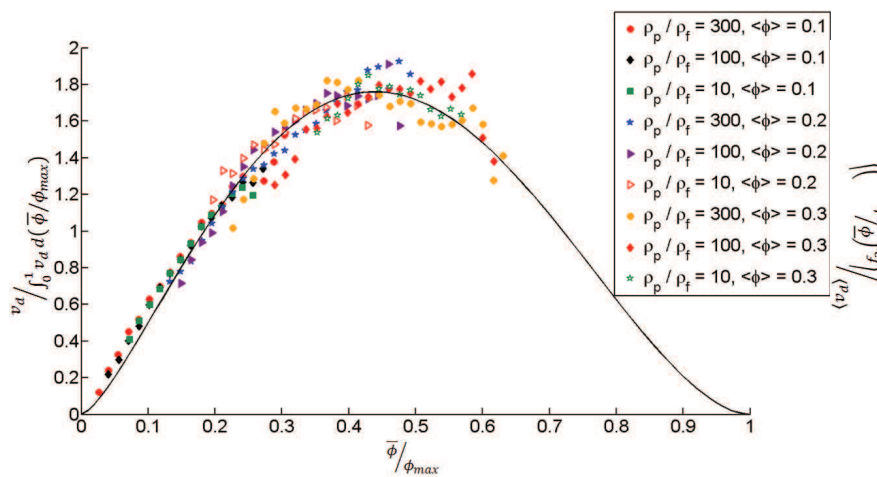

(a)

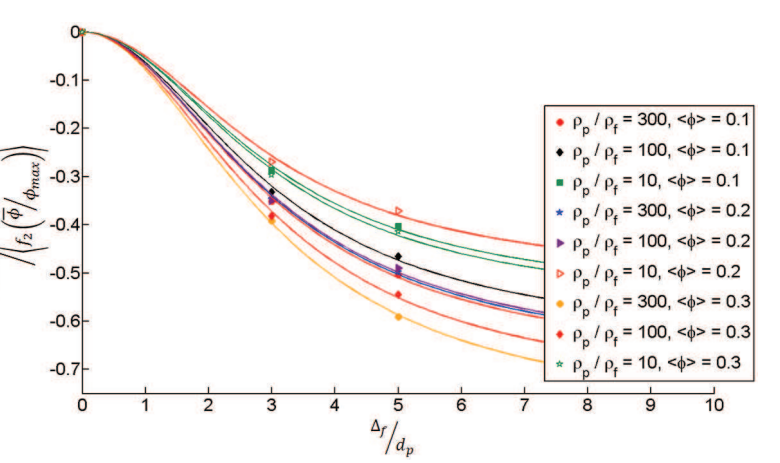

(b)

FiguRE 10. (a) The results for $v_{d}$, normalized by $\int_{0}^{1} v_{d} d\left(\bar{\phi} / \phi_{\max }\right)$, are shown as a function of $\bar{\phi} / \phi_{\max }$. These results are computed using $\Delta_{f} / d_{p}=3$. The results from the different particle configurations all fall onto a single curve, which we define as: $f_{2}\left(\bar{\phi} / \phi_{\max }\right)=\left(\bar{\phi} / \phi_{\max }\right)^{1.44}\left(1-\bar{\phi} / \phi_{\max }\right)^{1.84}$. (b) The domain-averaged drift flux results, $\left\langle v_{d}\right\rangle$, normalized by $\left\langle f_{2}\left(\bar{\phi} / \phi_{\max }\right)\right\rangle$, are shown as a function of $\Delta_{f} / d_{p}$. The results from the different particle configurations are all modeled using a single function, $\left\langle\kappa_{2}\right\rangle g_{2}\left(\Delta_{f} / d_{p}\right)=\left\langle\kappa_{2}\right\rangle \frac{\left(\Delta_{f} / d_{p}\right)^{2}}{9.20+\left(\Delta_{f} / d_{p}\right)^{2}}$, which provides the functional form for the different fitting curves. The results for both of these figures are compiled from fluidized bed simulations with the following set of parameters: $\rho_{p} / \rho_{f}=10,100$, and 300 and $\langle\phi\rangle=0.1,0.2$, and 0.3 .

In equation 3.18 , the parameter $k_{1}$ defines how quickly the mesh-dependence of $\overline{\left(\phi^{\prime}\right)^{2}}$ is saturated at higher filter sizes, with smaller values of $k_{1}$ correlating with this saturation occurring at lower values of $\Delta_{f}$. The current analysis has been performed using a domain size of $n_{x}=12 d_{p}$. In order to determine how this saturation parameter, $k_{1}$, varies with domain size, we have also performed this analysis at other domain sizes $\left(n_{x}=9 d_{p}\right.$ and $\left.24 d_{p}\right)$. From this analysis, it appears that as the domain size increases, the mesh-dependence of $\overline{\left(\phi^{\prime}\right)^{2}}$ becomes saturated at higher filter sizes, and so $k_{1}$ increases roughly as the square root of the domain size. Thus, as we look to apply this new drag constitutive relation to large-scale fluidized bed simulations, we can extrapolate this trend in order to conclude that: $k_{1} \gg\left(\Delta_{f} / d_{p}\right)^{2}$ for large-scale systems. Using this relationship, the scale-similar form for $\overline{\left(\phi^{\prime}\right)^{2}}$ can be written in its modified form as:

$$
\overline{\left(\phi^{\prime}\right)^{2}}=\kappa_{1}^{\prime} f_{1}\left(\bar{\phi} / \phi_{\max }\right) g_{1}^{\prime}\left(\Delta_{f} / d_{p}\right)
$$

where $\kappa_{1}^{\prime}=\kappa_{1} / k_{1}$ and $g_{1}^{\prime}\left(\Delta_{f} / d_{p}\right)=\left(\Delta_{f} / d_{p}\right)^{2}$.

\subsubsection{Drift flux}

Since the drift flux is a sub-grid-scale measure for the extent of inhomogeneities, we employ, as we did for $\overline{\left(\phi^{\prime}\right)^{2}}$ in section 3.5.1, a scale-similar method to estimate this quantity. A scalesimilar form for the dimensionless drift flux is given by:

$$
v_{d}=\kappa_{2} f_{2}\left(\bar{\phi} / \phi_{\max }\right) g_{2}\left(\Delta_{f} / d_{p}\right) .
$$

In order to determine a model for $f_{2}$, the dependence of $v_{d}$ on the concentration of particles, this function, as shown in figure 10a, is computed as:

$$
f_{2}\left(\bar{\phi} / \phi_{\max }\right)=\frac{v_{d}}{\int_{0}^{1} v_{d} d\left(\bar{\phi} / \phi_{\max }\right)} .
$$

Like $\overline{\left(\phi^{\prime}\right)^{2}}, v_{d}$ is taken to be 0 at the two $\bar{\phi} / \phi_{\max }$ limits. Using a filter size of $\Delta_{f} / d_{p}=3$, the results 
for $v_{d} / \int_{0}^{1} v_{d} d\left(\bar{\phi} / \phi_{\max }\right)$ are then computed as a function of $\bar{\phi} / \phi_{\max }$. The results in figure 10a are compiled from fluidized bed simulations with the following set of parameters: $\rho_{p} / \rho_{f}=10,100$, and 300 and $\langle\phi\rangle=0.1,0.2$, and 0.3 . From figure 10a, it is clear that the results from these different configurations all fall onto a single curve, with the following functional form:

$$
f_{2}\left(\bar{\phi} / \phi_{\max }\right)=\left(\bar{\phi} / \phi_{\max }\right)^{n_{2}}\left(1-\bar{\phi} / \phi_{\max }\right)^{m_{2}}
$$

where $n_{2}=1.44, m_{2}=1.84$, and $\phi_{\max }=0.64 . f_{2}\left(\bar{\phi} / \phi_{\max }\right)$ is valid over a range of length scales, as it has also been shown to hold at $\Delta_{f} / d_{p}=5$ to within $5 \%$. In addition, while figure 10 a provides only a sampling of the fluidized beds that were simulated in the current study, the universal shape of equation 3.25 holds over the full range of $\rho_{p} / \rho_{f}$ and $\langle\phi\rangle$ that were investigated. Furthermore, given the fact that the universal shape holds over the entire sampled $\bar{\phi}(0-0.4)$ and that prior multiphase flow studies employing a scale-similarity technique (Ozel et al. 2013) have found a similar concentration dependence, the universal shape of equation 3.25 should hold over the full range of $\bar{\phi}$.

Comparing the results of figure 10a with those of figure 9a, the collapse of the $v_{d}$ data points onto a single universal curve is found to be not as strong as that of $\overline{\left(\phi^{\prime}\right)^{2}}$, especially near the peak of the curve. Since $v_{d}$ involves both $\bar{\phi}$ and $\tilde{\boldsymbol{u}}_{\text {slip }}$, there is a possibility that attaining a universal shape for $v_{d}$ as a function of $\bar{\phi}$ is more difficult as compared to $\overline{\left(\phi^{\prime}\right)^{2}}$, which only involves $\bar{\phi}$. Near the peak of the curve in figure 10a, the overall effects of inhomogeneities are at their most extreme, so any additional effects of inhomogeneities in $\tilde{\boldsymbol{u}}_{\text {slip }}$ would further complicate the attempts to obtain a collapse of the $v_{d}$ data points onto a single curve. Still, based on the fact that the data points in figure 10a generally fall onto the universal curve, the scale-similar approach is still found to be appropriate for modeling $v_{d}$.

Next, in order to determine a model for $g_{2}$, the mesh-dependence of $v_{d}$, this function, as shown in figure 10b, is computed as:

$$
g_{2}\left(\Delta_{f} / d_{p}\right)=\frac{\left\langle v_{d}\right\rangle}{\left\langle\kappa_{2} f_{2}\left(\bar{\phi} / \phi_{\max }\right)\right\rangle},
$$

with $f_{2}$ defined by equation 3.25 . In figure $10 \mathrm{~b}$, the results for $\left\langle v_{d}\right\rangle /\left\langle f_{2}\left(\bar{\phi} / \phi_{\max }\right)\right\rangle$ are shown as a function of $\Delta_{f} / d_{p}$. The results for each different configuration have the same dependence on $\Delta_{f} / d_{p}$, and simply vary by the multiplicative constant, $\left\langle\kappa_{2}\right\rangle$. For all of these cases, $\left\langle\kappa_{2}\right\rangle<0$ because $v_{d}$ is typically negative over the entire fluidized bed, as explained in section 3.4.3. The functional dependence of $v_{d}$ on $\Delta_{f} / d_{p}$ is found to be:

$$
g_{2}\left(\Delta_{f} / d_{p}\right)=\frac{\left(\Delta_{f} / d_{p}\right)^{2}}{k_{2}+\left(\Delta_{f} / d_{p}\right)^{2}}
$$

where $k_{2}=9.20$. While larger computational domains are necessary in order to fully probe the validity of the quadratic mesh dependence in the smaller filter size regime, the form of equation 3.27 is confirmed in the current study through its ability to effectively pass through all of the data points in figure $10 \mathrm{~b}$ and by the fact that this equation is consistent with the Taylor series expansion of the drift velocity that is performed by Ozel et al. (2013). The curves, based on $\left\langle\kappa_{2}\right\rangle g_{2}\left(\Delta_{f} / d_{p}\right)$, are shown, in figure $10 \mathrm{~b}$, to fit the results over a range of system parameters. As was true for $\kappa_{1}$ in section 3.5.1, $\kappa_{2}$ is found to be larger for particle configurations taken from fluidized beds with larger values of $\rho_{p} / \rho_{f}$.

Using a test filter approach similar to the one described in equation 3.21 for the $\overline{\left(\phi^{\prime}\right)^{2}}$ measure, 
$\kappa_{2}$ can be estimated throughout the fluidized bed system as:

$$
\kappa_{2}=\frac{\widehat{\bar{\phi}\left|\widetilde{\boldsymbol{u}}_{\text {slip }}\right|}-\widehat{\bar{\phi}\left|\widetilde{\boldsymbol{u}}_{\text {slip }}\right|}}{f_{2}\left(\widehat{\bar{\phi}} / \phi_{\text {max }}\right) g_{2}\left(\Delta_{f, \text { test }} / d_{p}\right) \widetilde{\left|\widetilde{\boldsymbol{u}}_{\text {slip }}\right|}-\overline{f_{2}\left(\bar{\phi} / \phi_{\text {max }}\right) g_{2}\left(\Delta_{f} / d_{p}\right)\left|\widetilde{\boldsymbol{u}}_{\text {slip }}\right|}} .
$$

Thus, the dimensionless drift flux can be approximated through scale-similar modeling using equations $3.23,3.25,3.27$, and 3.28. In doing so, $v_{d}$ can be estimated in simulations where the flow is not fully resolved.

Analogous to the domain-size dependence of $k_{1}$, which is discussed in section $3.5 .1, k_{2}$ is found to increase with the domain size. Thus, the modified scale-similar model for the drift flux that is applicable to larger-scale simulations is given by:

$$
v_{d}=\kappa_{2}^{\prime} f_{2}\left(\bar{\phi} / \phi_{\max }\right) g_{2}^{\prime}\left(\Delta_{f} / d_{p}\right)
$$

where $\kappa_{2}^{\prime}=\kappa_{2} / k_{2}$ and $g_{2}^{\prime}\left(\Delta_{f} / d_{p}\right)=\left(\Delta_{f} / d_{p}\right)^{2}$.

Further details for implementing the inhomogeneity-dependent constitutive closures for the fluid-particle drag force can be found in the Supplementary Material.

\subsection{Error analysis of new drag models}

In order to conduct an analysis of these newly-developed drag models, the filtered $\left(\Delta_{f} / d_{p}=3\right)$, dimensionless drag, $F$, is computed via fully-resolved simulations in fluidized beds with $\rho_{p} / \rho_{f}=4,10,100,300,1500$, and 3000 and $\langle\phi\rangle=0.1,0.15,0.2,0.25$, and 0.3 , and these LBM drag values are compared with the drag that is predicted by these new constitutive models. These new drag models account for the effect of inhomogeneities by involving a sub-grid measure, either based on $\overline{\left(\phi^{\prime}\right)^{2}}$ or $v_{d}$. The $\overline{\left(\phi^{\prime}\right)^{2}}$-dependent model is defined in equation 3.9, while the $v_{d}$-dependent model is defined in equation 3.11 .

When using these new constitutive relations to predict the fluidized bed drag, we are able to evaluate the sub-grid-scale measure for the extent of inhomogeneities either by computing the quantity directly using the detailed flow information from the fully-resolved simulation (henceforth, referred to as a direct computation), or by estimating the quantity using the scalesimilar approach, detailed in section 3.5, with $\Delta_{f, t e s t} / d_{p}=5$. Since the drag relations will be applied as constitutive closures to larger-scale simulations, where the sub-grid-scale quantities cannot be directly computed, the latter analysis is a better test for how much improvement in the drag prediction we have achieved. However, the former analysis does allow us to ascertain how effective each of these inhomogeneity measures would be in predicting the drag if we were able to achieve a perfect estimation of these sub-grid quantities. The prediction errors of this drag model can be thought of as the best possible outcome if we are able to further improve our methodology for estimating the sub-grid quantities.

Since we have developed two different drag models that are dependent on $\overline{\left(\phi^{\prime}\right)^{2}}$ and $v_{d}$, respectively, and we are computing these sub-grid-scale quantities using two different approaches, we are essentially analyzing the performance of four different inhomogeneous drag models. In figure 11, in addition to analyzing the drag predictions of these four inhomogeneous models, we have included the results from the Rubinstein et al. (2016) drag model (same curve as in figure 4), so that we would have the St-dependent, homogeneous model for direct comparison. In figure 11 , the probability distribution function for each model's percent error in the drag prediction is plotted. The model bias, measured using both the prediction error at the PDF maximum and the average prediction error, and the spread in the errors for each of these five models are summarized in table 4. 


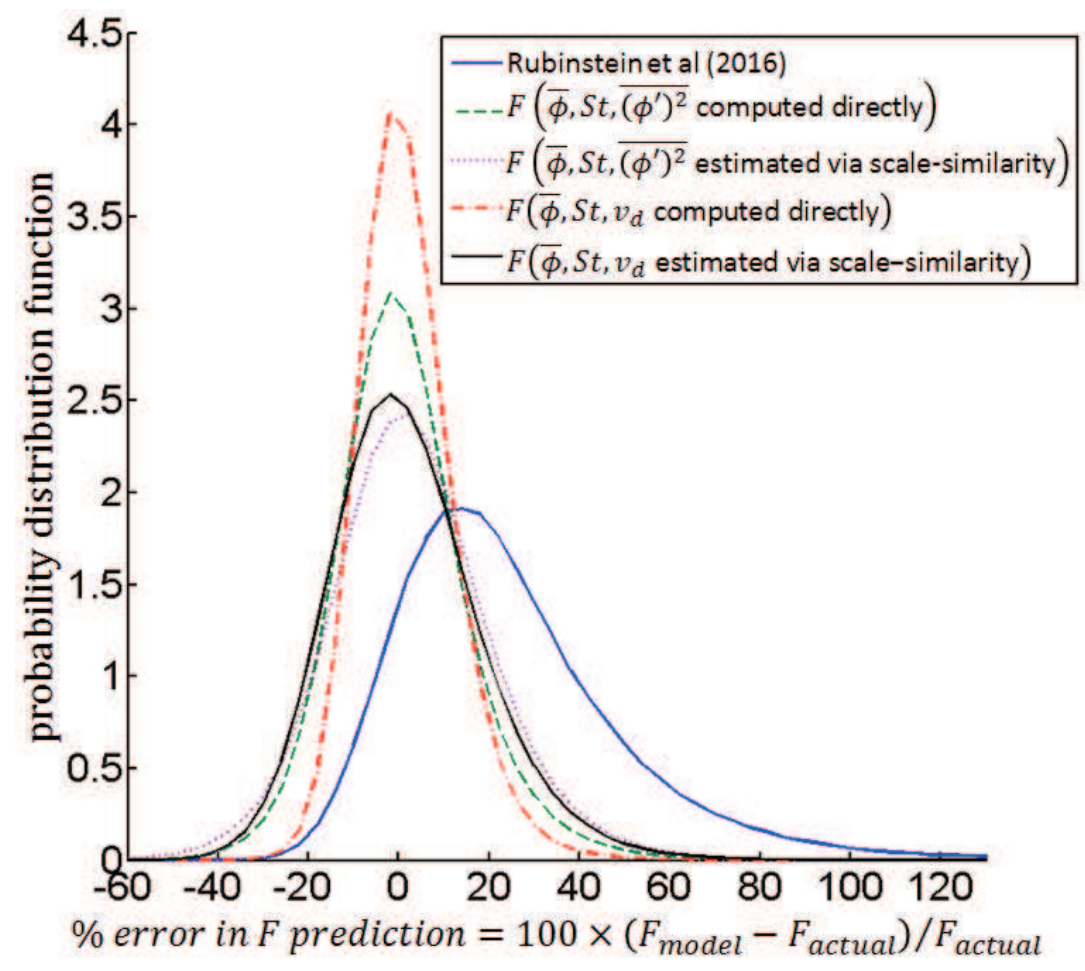

FIGURE 11. The probability distribution functions are plotted for the percent error in the drag prediction for five different drag models. The homogeneous drag model of Rubinstein et al. (2016) is compared to our new drag models that account for the effects of inhomogeneities. These drag models involve a sub-grid measure for the extent of inhomogeneities, either based on $\overline{\left(\phi^{\prime}\right)^{2}}$ or $v_{d}$. Each of these sub-grid-scale measures is either computed directly through a fully-resolved simulation, or estimated using a scale-similar method. These drag models are applied to fluidized bed systems with the following set of parameters: $\rho_{p} / \rho_{f}=4$, $10,100,300,1500$, and 3000 and $\langle\phi\rangle=0.1,0.15,0.2,0.25$, and 0.3 . This analysis is performed with $\Delta_{f} / d_{p}=3$ and $\Delta_{f, t e s t} / d_{p}=5$.

$$
\begin{gathered}
\text { Drag model } \\
\text { Rubinstein et al. (2016) } \\
F\left(\bar{\phi}, S t, \overline{\left(\phi^{\prime}\right)^{2}} \text { computed directly }\right) \\
F\left(\bar{\phi}, S t, \overline{\left(\phi^{\prime}\right)^{2}} \text { estimated via scale-similarity }\right) \\
F\left(\bar{\phi}, S t, v_{d}\right. \text { computed directly) } \\
F\left(\bar{\phi}, S t, v_{d} \text { estimated via scale-similarity }\right)
\end{gathered}
$$

\begin{tabular}{|c|}
\hline $\begin{array}{l}\text { Average } \\
\% \text { error } \\
24.0 \%\end{array}$ \\
\hline $1.0 \%$ \\
\hline $2.8 \%$ \\
\hline $1.8 \%$ \\
\hline
\end{tabular}

$\%$ Error at
PDF maximum
$14.0 \%$
$-2.0 \%$
$2.0 \%$
$-2.0 \%$
$-2.0 \%$

Spread in

$\%$ error

$22.8 \%$

$14.2 \%$

$18.5 \%$

$11.2 \%$

$17.9 \%$

TABLE 4 . The percent error at the maximum of the probability distribution functions, the average percent error, and spread in the distribution of the percent errors of one homogeneous and four inhomogeneous drag models are presented, when these models are applied, using $\Delta_{f} / d_{p}=3$ and $\Delta_{f, t e s t} / d_{p}=5$, to fluidized bed systems with the following set of parameters: $\rho_{p} / \rho_{f}=4,10,100,300,1500$, and 3000 and $\langle\phi\rangle=0.1,0.15$, $0.2,0.25$, and 0.3 .

From figure 11 and table 4, the fact that all four inhomogeneous drag models have a very small bias $( \pm 3.0 \%)$ confirms that we have properly fit the simulation data with these new models over a full range of $\bar{\phi}$ and St. While the error distributions are slightly skewed towards larger positive errors, this same conclusion can be made using either of the two measures for the model bias. In contrast, the Rubinstein et al. (2016) model, on average, over-predicts the drag by $24.0 \%$ due to the fact that this model was developed for homogeneous distributions of particles, and is therefore unable to account for the reduction in drag caused by inhomogeneities. 
Since all four inhomogeneous drag models have essentially zero bias when predicting the drag for this particular set of LBM data, the key parameter in determining the utility of these models is the spread in the errors. Out of the four inhomogeneous models, the drift flux-dependent drag model, in which the drift flux is computed directly, has the smallest spread in the percent error: $11.2 \%$. In comparison, the $\overline{\left(\phi^{\prime}\right)^{2}}$-dependent drag model, in which $\overline{\left(\phi^{\prime}\right)^{2}}$ is computed directly, has a spread in the percent error of $14.2 \%$. Thus, it is clear that if we are able to achieve a perfect estimation of these two sub-grid quantities, the drift flux measure appears to provide a better fit for the drag force than the $\overline{\left(\phi^{\prime}\right)^{2}}$ measure. From this analysis, it appears that incorporating the slip velocity into the measure for inhomogeneities provides a more powerful tool for modeling the drag than one that is solely based on the configuration of particles. Furthermore, in contrast with the homogeneous drag model of Rubinstein et al. (2016), both of these new drag models are able to have a significantly smaller spread due to their ability to account for the effect of inhomogeneities.

From figure 4 and table 1, we observe that there is a modest increase in the spread in the prediction error when the sub-grid quantities are estimated using a scale-similar approach with $\Delta_{f, t e s t} / d_{p}=5$, rather than through a direct computation. Given this observed reduction in the model accuracy, it is clear that the scale-similar approach for estimating the sub-grid quantities slightly degrades the quality of the drag force predictions. In particular, the fact that both models involving a scale-similar estimation have very similar spreads in the prediction error indicates that this effect is especially pronounced in the case of the drift flux scale-similar approach. Still, even when we use the scale-similar method to estimate the sub-filter quantities, the spreads in the error predictions are still significantly smaller than for the Rubinstein et al. (2016) case. This result further demonstrates that we have improved on the prior homogeneous drag models by accounting for the effect of sub-grid-scale inhomogeneities.

From the results of this study, it is clear that the $\overline{\left(\phi^{\prime}\right)^{2}}$ - and $v_{d}$-based drag models each have their relative advantages and disadvantages. One of the main advantages of the drift flux measure is that, as seen in figure 11, if these sub-grid-scale quantities can be directly computed, the $v_{d}$-dependent drag model provides a more precise representation of the actual drag than the $\overline{\left(\phi^{\prime}\right)^{2}}$-dependent drag model. On the other hand, one of the main advantages for $\overline{\left(\phi^{\prime}\right)^{2}}$ is that, as seen in figures $9 \mathrm{a}$ and $10 \mathrm{a}, \overline{\left(\phi^{\prime}\right)^{2}}$ more strongly fits the scale-similarity approximation than does the drift flux. Due to this difference in the applicability of the scale-similar approach, in figure 11, the two inhomogeneous drag models are found to have almost identical precision when the sub-grid-scale quantities are estimated using scale-similarity. In addition, $v_{d}$ is not only based on the configurations of particles, but also on the flow structures, which allows the drift flux to account for inhomogeneities in the slip velocity, whereas $\overline{\left(\phi^{\prime}\right)^{2}}$ is only able to account for inhomogeneities in the particle distribution. However, $\overline{\left(\phi^{\prime}\right)^{2}}$ has a simpler physical interpretation, as it is simply a measure of the fluctuations in the particle volume fraction, while the drift flux is a measure of the correlation between the slip velocity and the particle volume fraction. Based on this analysis, neither one of these options is definitively the better one, and so, instead, each of these two measures for the extent of sub-grid-scale inhomogeneities should be considered in future studies of the effects of inhomogeneities on the drag.

Based on the observed increase in the spread of the drag prediction errors when employing the scale-similar approach, we see that there is some room for improvement in our modeling of the sub-grid-scale inhomogeneities. One alternate technique for estimating these sub-grid quantities is through the use of additional transport equations. Since these transport equations require additional constitutive closures, depending on the quality of these closures, this approach has the potential to improve the precision of our sub-grid quantity estimation. Overall, the current 
work provides a promising approach for accounting for the effect of inhomogeneities on the fluidparticle drag force that can be further improved upon in future studies.

\section{Summary}

Through this work, using LBM simulations of fluidized beds with a cubic periodic domain analyzed over a range of filter sizes, the effect of inhomogeneities on the fluid-particle drag force is quantified in the low $R e$ regime through one of two measures: the scalar variance of the particle volume fraction, $\overline{\left(\phi^{\prime}\right)^{2}}$, or the dimensionless drift flux, $v_{d}$. Two new drag models (equations 3.9 and 3.11) are then proposed that are, in addition to being functions of $\bar{\phi}$ and $S t$, dependent on one of these two measures for the extent of inhomogeneities. Unlike prior drag models that were derived under the assumption that the configuration of particles is randomly homogeneous, the newly proposed constitutive relations are able to account for the significant reduction in drag that occurs in the low $R e$ regime due to the presence of inhomogeneities.

By focusing on the low $R e$ regime, the current study is able to investigate the effects of inhomogeneities without the additional influences of inertial forces and granular temperature, which play a significant role at higher $R e$. A typical low $R e$ fluidized bed is one that is fluidized by ambient air, and whose particles have a diameter of $75 \mu \mathrm{m}$ and a density of $1.5 \mathrm{~g} / \mathrm{cm}^{3}$. While the terminal velocity of these particles is $\sim 20 \mathrm{~cm} / \mathrm{s}$, the minimum fluidization velocity is only about $4 \mathrm{~mm} / \mathrm{s}$ and the minimum bubbling velocity is about $2 \mathrm{~cm} / \mathrm{s}$, which are both within the low $R e$ regime. While low $R e$ fluidized beds can exhibit both non-bubbling and bubbling behavior, industrial fluidization processes typically involve faster flow velocities, and are therefore in the moderate-to-high $R e$ regime. However, even in industrial fluidized beds, the local fluid-particle slip velocities that govern the drag force are considerably smaller than the overall gas flow velocities. Having established the effects of inhomogeneities on the drag in the low Re limit, the approach used in the current study can be utilized to extend this analysis to systems with higher $R e$. Higher Re studies would provide insights into how the interplay between inhomogeneities, which tend to reduce the drag, and inertial forces and granular temperature, which tend to increase the drag, combine to affect the overall interactions between the fluid and particles.

The two identified measures for the extent of inhomogeneities, $\overline{\left(\phi^{\prime}\right)^{2}}$ and $v_{d}$, in our new drag models are both sub-grid quantities. In contrast, the gradient in the volume fraction, $\nabla \bar{\phi}$, which was shown in prior studies to be a useful measure for the extent of inhomogeneities, is found to inadequately model the drag over a range of length scales due to its inability to account for subgrid-scale inhomogeneous structures. Thus, we have observed that the effect of inhomogeneities on the drag force are primarily tied to the extent of sub-grid-scale inhomogeneities, as these are the structures that are not resolved at the scale at which the drag is computed.

An approach for estimating the sub-grid measures is needed in order to utilize these sub-gridquantity-dependent drag relations in larger-scale simulations of fluidized beds, where the flow is not fully resolved. While the use of additional transport equations has the potential to provide higher accuracy in estimating these sub-grid quantities, in this study, the sub-grid quantities are estimated using a scale-similar approach, in which, based on the structures observed at a second, larger test filter size, the measure for the extent of inhomogeneities is extrapolated down to the $\Delta_{f}$-length scale. The scale-similar models for $\overline{\left(\phi^{\prime}\right)^{2}}$ and $v_{d}$ are described by equations 3.14 and 3.23, respectively. Using these scale-similar approaches to estimate the sub-filter quantities, both of the new drag models are shown to provide an improvement in the precision of the drag predictions over the prior homogeneous drag models.

While a direct quantitative comparison between the results in the current study and those of 
prior inhomogeneity studies cannot be made due to the fact these prior studies have not resulted in a generalized, low $R e$ constitutive drag relation, the key observed trends can, however, be compared. In these prior works (Zhou et al. 2014; Li et al. 2016), the fluid-particle drag force is found to depend on both the direction and magnitude of the particle volume fraction gradient, with volume fraction gradients in the direction of the fluid-particle slip velocity causing an increase in drag, and volume fraction gradients perpendicular to the slip velocity causing a decrease in drag. In the current work, the drag force is found to depend only on the magnitude of the inhomogeneities, with increases in the inhomogeneities leading to a decrease in drag. Putting the results of the current work in the context of these prior studies, the effects of the volume fraction gradients perpendicular to the slip velocity significantly outweigh those of the parallel components. Overall, all of these inhomogeneity studies have identified the significant limitations in homogeneous drag relations due to their inability to account for inhomogeneities. The current work, however, is the first to propose a robust drag model that accounts for the effects of inhomogeneities over a full range of low $R e$, dynamic fluidized beds.

In order to fully assess the effect of incorporating measures for the extent of inhomogeneities within the new drag constitutive relations, these new drag models must next be applied to larger-scale simulations of fluidized beds. In doing so, the differences between the fluidized bed behavior predicted using these new drag relations and that using the prior homogeneous drag models will be ascertained, especially in relation to the grid resolution at which convergence in the flow behavior is achieved, and to the flow structures that develop as a result of the fluid-particle interactions. Based on the strong effect of inhomogeneities on the fluid-particle drag force that we have demonstrated over the course of this study, the newly proposed drag models should provide a significant improvement over prior constitutive relations that fail to account for the extent of inhomogeneities.

This work is supported by a grant from the ExxonMobil Research \& Engineering Co., and by a fellowship awarded to G.J.R. by the National Science Foundation (DGE-1148900).

\section{REFERENCES}

Agrawal, K., Loezos, P. N., Syamlal, M. \& Sundaresan, S. 2001 The role of meso-scale structures in rapid gas-solid flows. J. Fluid Mech. 445, 151-185.

Beetstra, R., van Der Hoef, M. A. \& Kuipers, J. A. M. 2007 Drag Force of Intermediate Reynolds Number Flow Past Mono- and Bidisperse Arrays of Spheres. AIChE J. 52 (2), 489-501.

ten Cate, A., Nieuwstad, C. H., Derksen, J. J. \& van den AkKer, H. E. A. 2002 Particle imaging velocimetry experiments and lattice-Boltzmann simulations on a single sphere settling under gravity. Phys. Fluids 14 (11), 4012-4025.

Chen, S. \& Doolen, G. D. 1998 Lattice Boltzmann method for fluid flows. Annu. Rev. Fluid Mech. 30, 329-364.

Cloete, S., Johansen, S. T. \& Amini, S. 2015 Grid independence behaviour of fluidized bed reactor simulations using the Two Fluid Model: Effect of particle size. Powder Technol. 269, 153-165.

DERKSEN, J. \& VAN DEN AKKER, H. E. A. 1999 Large-eddy simulations on the flow driven by a Rushton turbine. AIChE J. 45, 209-221.

Derksen, J. J. \& Sundaresan, S. 2007 Direct numerical simulations of dense suspensions: wave instabilities in liquid-fluidized beds. J. Fluid Mech. 587, 303-336.

Eggels, J. G. M. \& Somers, J. A. 1995 Numerical simulation of free convective flow using the latticeBoltzmann scheme. Int. J. Heat Fluid Flow 16 (5), 357-364.

Fox, R. O. 2014 On multiphase turbulence models for collisional fluidparticle flows. J. Fluid Mech. $\mathbf{7 4 2}$, 368-424.

Fullmer, W. D. \& HRenya, C. M. 2016 Quantitative assessment of fine-grid kinetic-theory-based predictions of mean-slip in unbounded fluidization. AIChE J. 62 (1), 11-17. 
Garside, J. \& AL-Dibouni, M. R. 1977 Velocity-Voidage Relationships for Fluidization and Sedimentation in Solid-Liquid Systems. Ind. Eng. Chem. Process Des. Dev. 16, $206-214$.

Germano, M., Piomelli, U., Moin, P. \& CAвот, W. H 1991 A dynamic subgrid-scale eddy viscosity model. Phys. Fluids A-Fluid 3 (7), 1760-1765.

Goldstein, D., Handler, R. \& Sirovich, L. 1993 Modeling a No-Slip Flow Boundary with an External Force Field. J. Comput. Phys. 105 (2), 354-366.

HILl, R. J., KOCH, D. L. \& LADD, A. J. C. 2001 The first effects of fluid inertia on flows in ordered and random arrays of spheres. J. Fluid Mech. 448, 213-241.

van der Hoef, M. A., Beetstra, R. \& Kuipers, J. A. M. 2005 Lattice-Boltzmann simulations of low-Reynolds-number flow past mono- and bidisperse arrays of spheres: results for the permeability and drag force. J. Fluid Mech. 528, 233-254.

Hong, K., Chen, S., WANG, W. \& LI, J. 2016 Fine-grid two-fluid modeling of fluidization of Geldart A particles. Powder Technol. 296, 2-16.

IgCi, Y., Andrews IV, A. T., Sundaresan, S., Pannala, S. \& O’Brien, T. 2008 Filtered two-fluid models for fluidized gas-particle suspensions. AIChE J. 54 (6), 1431-1448.

IGCI, Y. \& SundaRESAN, S. 2011 Constitutive models for filtered two-fluid models of fluidized gasparticle flows. Ind. Eng. Chem. Res. 50, 13190-13201.

Jiménez, C., Ducros, F., Cuenot, B. \& Bédat, B. 2001 Subgrid scale variance and dissipation of a scalar field in large eddy simulations. Phys. Fluids 13 (6), 1748-1754.

Kim, S. \& KARIlla, S. J. 1991 Microhydrodynamics: Principles and Selected Applications. Boston: Butterworth-Heinemann.

Kriebitzsch, S. H. L., van der Hoef, M. A. \& Kuipers, J. A. M. 2013 Drag force in discrete particle models-Continuum scale or single particle scale? AIChE J. 59 (1), 316-324.

LADD, A. J. C. 1994 Numerical simulations of particulate suspensions via a discretized Boltzmann equation. Part 1. Theoretical foundation. J. Fluid Mech. 271, 285-309.

Li, T., Gel, A., Pannala, S., Shahnam, M. \& Syamlal, M. 2014 Reprint of "CFD simulations of circulating fluidized bed risers, part I: Grid study". Powder Technol. 265, 2-12.

Li, T., WAng, L., Rogers, W., Zhou, G. \& GE, W. 2016 An approach for drag correction based on the local heterogeneity for gas-solid flows. AIChE J. .

Liu, X., Zhu, C., Geng, S., Yao, M., Zhan, J. \& Xu, G. 2015 Two-fluid modeling of Geldart A particles in gas-solid micro-fluidized beds. Particuology 21, 118-127.

LU, B., WANG, W. \& LI, J. 2009 Searching for a mesh-independent sub-grid model for CFD simulation of gas-solid riser flows. Chem. Eng. Sci. 64 (15), 3437-3447.

NGuyen, N.-Q. \& LAdD, A. J. C. 2002 Lubrication corrections for lattice-Boltzmann simulations of particle suspensions. Phys. Rev. E. 66, 046708.

Ozel, A., Fede, P. \& Simonin, O. 2013 Development of filtered Euler-Euler two-phase model for circulating fluidised bed: High resolution simulation, formulation and a priori analyses. Int. J. Multiph. Flow 55, 43-63.

Ozel, A., Parmentier, J. F., Simonin, O. \& Fede, P. 2010 A priori test of effective drag modeling for filtered two-fluid model simulation of circulating and dense gas-solid fluidized beds. In 7th International Conference on Multiphase Flow-ICMF.

PARMentier, J.-F., Simonin, O. \& Delsart, O. 2012 A functional subgrid drift velocity model for filtered drag prediction in dense fluidized bed. AIChE J. 58 (4), 1084-1098.

PePiot, P. \& Desjardins, O. 2012 Numerical analysis of the dynamics of two- and three-dimensional fluidized bed reactors using an Euler-Lagrange approach. Powder Tech. 220, 104-121.

Qian, Y. H., D'Humieres, D. \& LALlemand, P. 1992 Lattice BGK for the Navier-Stokes equations. Eur. Lett. 17, 479-484.

Richardson, J. F. \& ZAKI, W. N. 1954 Sedimentation and fluidisation: Part I. Trans. Inst. Chem. Eng. 32, 35-53.

Rubinstein, G. J., Derksen, J. J. \& Sundaresan, S. 2016 Lattice Boltzmann simulations of lowReynolds-number flow past fluidized spheres: effect of Stokes number on drag force. J. Fluid Mech. 788, 576-601.

SCHNeIderbauer, S. \& PirkeR, S. 2014 Filtered and heterogeneity-based subgrid modifications for gas-solid drag and solid stresses in bubbling fluidized beds. AIChE J. 60 (3), 839-854.

Somers, J. A. 1993 Direct simulation of fluid flow with cellular automata and the lattice-Boltzmann equation. Appl. Sci. Res. 51 (1-2), 127-133. 
Tang, Y., Peters, E. A. J. F. \& Kuipers, J. A. M. 2016 Direct numerical simulations of dynamic gas-solid suspensions. AIChE J. 62 (6), 1958-1969.

Tenneti, S., Garg, R. \& Subramaniam, S. 2011 Drag law for monodisperse gas-solid systems using particle-resolved direct numerical simulation of flow past fixed assemblies of spheres. Int. J. Multiph. Flow 37, 1072-1092.

WAng, J., VAn Der Hoef, M. A. \& Kuipers, J. A. M. 2009 Why the two-fluid model fails to predict the bed expansion characteristics of Geldart A particles in gas-fluidized beds: A tentative answer. Chem. Eng. Sci. 64 (3), 622-625.

WANG, X., LiU, K. \& You, C. 2011 Drag force model corrections based on nonuniform particle distributions in multi-particle systems. Powder Technol. 209, 112-118.

Wen, C. Y. \& YU, Y. H. 1966 Mechanics of fluidization. Chem. Eng. Progress, Symp. Ser. 62 (1), 100111.

XU, M., GE, W. \& LI, J. 2007 A discrete particle model for particle-fluid flow with considerations of sub-grid structures. Chem. Eng. Sci. 62 (8), 2302-2308.

Zhou, G., Xiong, Q., Wang, L., Wang, X., Ren, X. \& Ge, W. 2014 Structure-dependent drag in gas-solid flows studied with direct numerical simulation. Chem. Eng. Sci. 116, 9-22. 